\title{
Numerical study of high speed evaporating sprays
}

\author{
6 Q1 Abolfazl Irannejad, Farhad Jaberi* \\ 7 Q2 Department of Mechanical Engineering, Michigan State University, East Lansing, MI 48824, United States
}

\section{A R T I C L E I N F O}

Article history:

Received 25 February 2014

Received in revised form 16 November 2014 Accepted 23 November 2014

Available online $\mathrm{xxxx}$

\section{Keywords:}

LES of multiphase flows

High injection pressure sprays

Spray-turbulence interactions

\begin{abstract}
A B S T R A C T
Large eddy simulations of high speed evaporating sprays are conducted to study spray interactions with the gas flow and turbulence generated by the spray. The spray is simulated with a Lagrangian droplet method and a stochastic breakup model together with non-equilibrium, finite-rate heat and mass transfer models. The Lagrangian spray/droplet field is fully coupled with the Eulerian gas flow through mass, momentum and energy coupling terms. The interaction of spray induced gas flow and turbulence with the droplets is studied for different gas chamber densities and temperatures as well as different nozzle sizes and injection pressures. Our results indicate that although the droplet transport and evaporation are both important to the generated gas flow and its interactions with the spray, the major source of momentum transfer to the gas is the high speed vapor generated by evaporation. It is shown that sprays injected from larger nozzles generate more perturbations in the gas due to increase in evaporation rate by higher entrained gas. However, the liquid spray penetration remains unchanged with the variation in injection pressure due to competing effects of evaporation and vapor convection. While the liquid penetration is not significantly affected by the injection pressure, the evaporated vapor penetrates more and mixes better at higher injection pressures due to higher induced gas velocity and turbulence.
\end{abstract}

(c) 2014 Published by Elsevier Ltd.

\section{Introduction}

High speed evaporating sprays and their two-way mass, momentum and energy interactions with the gas flow turbulence are important in advanced combustion systems. The mass and volume of the dispersed liquid with respect to the carrier gas are two of the critical parameters that determine the level of interaction between phases. Flows containing very small (in comparison to the Kolmogorov scale) droplets with small mass and volume fraction have gas flow and turbulence structure similar to that of a single-phase flow. However, with larger droplets and higher volume fractions, the liquid effects on gas turbulence production, dissipation and stresses become important (Crowe et al., 1998; Q3 Sirignano, 2010; Balachandar and Eaton, 2010). This, of course, depends on the spray speed and the level of background gas turbulence. When a spray is injected with low to moderate speeds into a quiescent chamber, the gas flow and turbulence stay insignificant. However, at high injection pressures and for high speed sprays (order of hundreds of meter per second), spray droplets can become a significant source of gas turbulence. This is partly due to drag of liquid droplets but can also be caused by the droplet evaporation as shown below. Balachandar and Eaton (2010) considered the main mechanisms for flow/turbulence modifica-

\footnotetext{
* Corresponding author.

E-mail address: jaberi@msu.edu (F. Jaberi).
}

tions by non-evaporating droplets to be: (i) the drag of larger droplets causing enhanced dissipation, (ii) the transfer of droplet kinetic energy to the gas, (iii) the wakes and vortex shedding behind large droplets and (iv) the buoyancy induced instabilities due to density variations arising from the preferential concentration of droplets. Depending on the ratio of the dispersed phase response time to the flow time scale, known as the Stokes number, $S t$, the dispersed phase particles change both the dissipation and the production of carrier fluid turbulence. For sprays issuing into a quiescent chamber with high injection pressures, the generated droplets have high velocities and large St numbers. Consequently, the droplet wake and enhanced dissipation mechanisms play lesser roles and the turbulence is mainly generated and modified by the droplet drag and the evaporation as explained below.

Two general types of computational models have been developed for numerical simulations of two-phase flows involving sprays (Balachandar and Eaton, 2010; Prosperetti and Tryggvason, 2007; Subramaniam, 2013). In the Eulerian-Lagrangian models, the continuous carrier phase is described by the Navier-Stokes equations on Eulerian grid points, while the spray is represented by discrete droplets, which are injected and tracked in the computational domain in a Lagrangian manner. The two-fluid Eulerian-Eulerian models treat the carrier fluid and the dispersed phase as "interpenetrating media" which are described by a set of mass, momentum and energy conservation equations. The Eulerian-Eulerian models assume the existence of unique field 
representations for particle velocity and temperature, implicitly restricting the maximum St number that can be considered (Balachandar and Eaton, 2010). The primary jet breakup in sprays can be simulated by Eulerian-Eulerian models (Gorokhovski and Herrmann, 2008; Shen and Yue, 2001; Li and Jaberi, 2009; Herrmann, 2010). These types of models are expected to better capture the main liquid jet breakup, but they are not currently practical and cannot be employed for high speed evaporating sprays in realistic systems. Far enough from the injector nozzle, the liquid droplets are small, dispersed and far from being a continuum. Consequently, they can be better represented by a collection of Lagrangian particles and Eulerian-Lagrangian models.

The method we refer to as the direct numerical simulation (DNS) can capture the two-way interactions of particles with the carrier turbulent flow. However, DNS is extremely demanding and currently impractical for high speed turbulent sprays. Large eddy simulation (LES) method on the other hand has been widely applied to multiphase flows and can be a viable tool for spray simulations (Fox, 2012), even though submodels are required in LES to account for various physical processes taking place at small or sugrid time and length scales in both Eulerian and Lagrangian fields. LES models have been recently applied to complex systems involving evaporating/reacting sprays and complex droplet-turbulence interactions at resolved and subgrid scale (SGS) levels (Moin and Apte, 2006; Patel and Menon, 2008; Banaeizadeh et al., 2013; Bini and Jones, 2009; Bharadwaj et al., 2009; Irannejad and Jaberi, 2014; Irannejad et al., in press). A number of spray LES studies have been focused on flows in complicated geometries (Moin and Apte, 2006; Patel and Menon, 2008; Banaeizadeh et al., 2013), while others discussed various SGS modeling issues. Bini and Jones (2009) indicated the importance of SGS models for evaporating sprays. It was pointed out that the neglect of SGS acceleration and vaporization of droplets causes poor predictions of the spray and the carrier fluid flow. Patel and Menon (2008) and Bharadwaj et al. (2009) included the subgrid spray drag force fluctuations in the SGS kinetic energy equation. Irannejad and Jaberi (2014) included the added mass contribution to the carrier gas kinetic energy at the subgrid level. They also considered other physical phenomena that affects the spray behavior such as the modification of droplet dynamics by wake interactions and the transient heating of droplets' interior in their LES calculations. Following this work, we use a similar LES/spray model in the present paper to study the physical behavior of turbulent flows generated by high speed evaporating sprays and the interactions that these flows have with the droplets under variety of flow and spray operating conditions.

Turbulence interactions with dispersed particles or droplets have been extensively studied in the past (Balachandar and Eaton, 2010; Hetsroni, 1989; Elghobashi and Truesdell, 1993; Mashayek and Pandya, 2003). Most of these studies were on the modulation of turbulence by particles (Crowe, 2000; Eaton, 2006; Eaton, 2009). However, some studies also considered the turbulence generation by the dispersed phase (Chen et al., 2000). Earlier numerical studies were focused on the isothermal and non-evaporating particle-laden flows. In flows with evaporating particles, additional complexities due to combined effects of interphase heat and mass transfers and turbulent mixing of particle vapor arise. Birouk and Gokalp (2006) reviewed studies conducted on the effect of turbulence on individual droplets. Most of these studies show that with increasing the turbulence level, the evaporation rate is increased. However, in an evaporating spray, droplets can significantly change the vapor mass fraction and other flow variables around droplets, which can in turn change the droplet evaporation. Reveillon and Demoulin (2007) in their DNS study, investigated the one way interaction of a forced isotropic turbulent flow with evaporating droplets at different Stokes numbers $(S t=0.17,1.05$ and 5.6) and found very different behavior. It was shown that droplet clusters are created because of the turbulence, leading to local vapor concentration and in turn slowing down of the droplet vaporization rate. The standard deviations of vapor concentration fluctuations, which were mostly attributed to the presence of droplet clusters, were strongly dependent on the Stokes number. On the other hand, there have been several studies on the two-way interactions of evaporating droplets with the gas turbulence. DNS and LES studies (Mashayek, 1998; Miller and Bellan, 1999; Okongo and Bellan, 2004; Leboissetier et al., 2005) for droplet-laden homogeneous shear and temporal shear layer flows, for example, showed that evaporation increases the turbulent kinetic energy and the dissipation of turbulent kinetic energy of the carrier gas. However, the majority of previous studies were focused on mono-dispersed droplets with low to moderate droplet Stokes numbers $(S t \leq 1)$. Experimental studies indicate that poly-dispersity of droplets can cause significant fluctuations in vapor concentration in highly turbulent flows (Cochet et al., 2009). Presently, our understanding of high speed poly-dispersed evaporating sprays is somewhat limited due to droplet poly-dispersity, interphase heat and mass transfers and complex interactions of high Stokes number $(S t \gg 1)$ droplets with the gas flow and turbulence.

This paper is on the detailed study of gas flow mass, momentum and energy interactions with very high speed evaporating droplets in sprays. The focus is on the flow and turbulence generated by the spray in the gas and the effects that they have on the spray/droplet evolution. Details of the computational model and the sensitivity of numerical results to various spray and LES submodels are discussed in our previous paper (Irannejad and Jaberi, 2014). The intent here in this paper is to better understand the complicated processes involved in high pressure sprays injected in high temperature and pressure chambers. Our results below show the importance and the complexity of the flow and turbulence generated by the spray.

\section{Mathematical formulation and numerical solution}

The two-phase LES model used in this work has two main mathematical components: (1) the Eulerian gas conservation equations, and (2) the Lagrangian spray equations. Fig. 1 shows various submodels embodied in the two components of LES/spray solver. As shown in this figure, for the carrier gas velocity, pressure and scalars we solve standard (Eulerian) filtered equations with appropriate SGS models. The spatial discretization of the carrier gas equations is based on the fourth-order compact finite difference scheme and the time differencing is based on a third order low storage Runge-Kutta method (Banaeizadeh et al., 2013; Irannejad and Jaberi, 2014; Afshari et al., 2008). The subgrid turbulence is modeled by a SGS kinetic energy equation that includes the spray effects on the SGS turbulence. The spray is simulated with a non-equilibrium Lagrangian particle method. The effect of SGS turbulence on the droplet is included through a stochastic subgrid model. The modification of droplet dynamics by wakes of nearby droplets is also considered. The spray breakup is modeled by a stochastic equation for the droplet size distribution. Heat and mass transfers between phases are calculated by solving mass and energy conservation equations for the droplet interior and surface. The Lagrangian spray equations are fully coupled with the Eulerian gas equations through mass, momentum, energy and species source/sink terms. The two components of the LES/spray model are further described in the following sections. More details can be found in Ref. (Irannejad and Jaberi, 2014).

\section{Eulerian filtered gas equations}

The gas phase compressible Favre-filtered continuity, momentum, energy and species mass fraction (scalar) equations are 


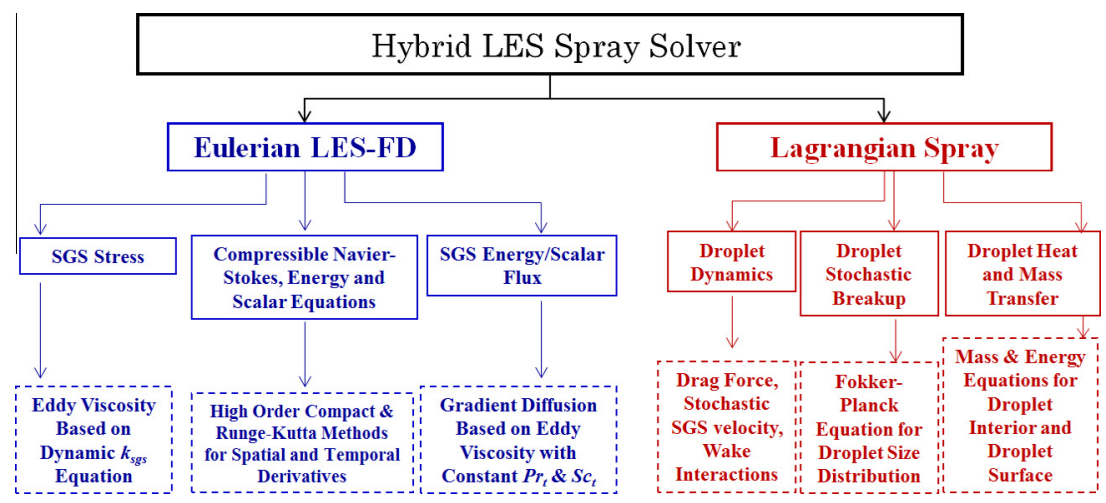

Fig. 1. Various submodels in the hybrid LES/Spray solver.

$$
\frac{\partial\langle\rho\rangle_{l}\left\langle u_{i}\right\rangle_{L}}{\partial t}+\frac{\partial\langle\rho\rangle_{l}\left\langle u_{i}\right\rangle_{L}\left\langle u_{j}\right\rangle_{L}}{\partial x_{j}}=-\frac{\partial\langle p\rangle_{l}}{\partial x_{i}}+\frac{\partial\left\langle\tau_{i j}\right\rangle_{l}}{\partial x_{j}}-\frac{\partial\left(\tau_{i j}^{s g s}\right)}{\partial x_{j}}+\left\langle\dot{S}_{m i}^{s p}\right\rangle_{l},
$$

$$
\begin{aligned}
& \frac{\partial\langle\rho\rangle_{l}\langle E\rangle_{L}}{\partial t}+\frac{\partial\left(\langle\rho\rangle_{l}\langle E\rangle_{L}+\langle p\rangle_{l}\right)\left\langle u_{i}\right\rangle_{L}}{\partial x_{i}} \\
& \quad=-\frac{\partial\left(\left\langle q_{i}\right\rangle_{l}+H_{i}^{s g s}\right)}{\partial x_{i}}+\left\langle\dot{S}_{E}^{s p}\right\rangle_{l}+\frac{\partial\left(\left\langle\tau_{i j}\right\rangle_{l}-\tau_{i j}^{s g s}\right)\left\langle u_{j}\right\rangle_{L}}{\partial x_{i}}
\end{aligned}
$$

$$
\frac{\partial\langle\rho\rangle_{l}\left\langle\phi_{\alpha}\right\rangle_{L}}{\partial t}+\frac{\partial\langle\rho\rangle_{l}\left\langle u_{i}\right\rangle_{L}\left\langle\phi_{\alpha}\right\rangle_{L}}{\partial x_{i}}=-\frac{\partial\left(\left\langle J_{i}^{\alpha}\right\rangle_{l}+J_{i}^{\alpha s g s}\right)}{\partial x_{i}}+\left\langle\dot{S}_{\phi}^{s p}\right\rangle_{l},
$$

where \langle\rangle$_{l}$ and \langle\rangle$_{L}$ represent the filtering and the Favre-filtering operators, respectively. The primary variables in Eqs. (1)-(4) are the filtered density, $\langle\rho\rangle_{l}$, the Favre-filtered velocity, $\left\langle u_{i}\right\rangle_{L}$, the Favrefiltered total energy, $\langle E\rangle_{L}=\langle e\rangle_{L}+\frac{1}{2}\left\langle u_{i}\right\rangle_{L}\left\langle u_{i}\right\rangle_{L}$ and the Favre-filtered species mass fraction, $\left\langle\phi_{\alpha}\right\rangle_{L}$. The filtered viscous stress tensor, $\left\langle\tau_{i j}\right\rangle_{l}$ is assumed to be a linear function of the Favre-filtered strain rate, $\left\langle S_{i j}\right\rangle_{L}$ for the simulated Newtonian fluid, and the filtered heat flux, $\left\langle q_{i}\right\rangle_{l}$ and species diffusion, $\left\langle J_{i}^{\alpha}\right\rangle_{l}$ vectors are evaluated based on Fourier's and Fick's laws. The filtered form of the ideal equation of state for gas, $\langle p\rangle_{l}=\langle\rho\rangle_{l}\langle R\rangle_{L}\langle T\rangle_{L}$ relates filtered density and pressure to the Favre- filtered temperature, $\langle T\rangle_{L}$ through mixture gas constant, $\langle R\rangle_{L}$. The mixture gas constant is calculated based on universal gas constant and the filtered mass fraction of species and their molecular weights.

\section{SubGrid scale models}

The unclosed SGS terms in the filtered equations are closed here by gradient type closures (Moin et al., 1991; Ghosal et al., 1995). In these closures, the characteristic length and velocity for defining the eddy viscosity are provided by the local grid size $\bar{\Delta}$ and the subgrid kinetic energy, $k_{s g s}$, as $v_{t}=c_{v} \bar{\Delta} \sqrt{k_{s g s}}$. The model coefficients are given in Table 1 . The subgrid kinetic energy is obtained by the solution of the following equation using dynamic coefficients and spray mass and momentum coupling terms (Irannejad and Jaberi, 2014).

Table 1

Subgrid-scale model coefficients in the Eulerian gas equations.

\begin{tabular}{lll}
\hline Coefficient & Model & Values \\
\hline$c_{v}, c_{k}$ & Eddy viscosity and SGS transport of & Dynamically \\
& $k_{\text {sgs }}$ & calculated \\
$c_{\epsilon}$ & Dissipation of $k_{s g s}$ & 1.0 \\
$P r_{t}, S c_{t}$ & SGS energy and scalar transport & 0.5 \\
\hline
\end{tabular}

$$
\begin{aligned}
\frac{\partial\langle\rho\rangle_{l} k_{s g s}}{\partial t}+\frac{\partial\langle\rho\rangle_{l}\left\langle u_{i}\right\rangle_{L} k_{s g s}}{\partial x_{i}}= & -\tau_{i j}^{s g s}\left\langle S_{i j}\right\rangle_{L}-c_{\epsilon}\langle\rho\rangle_{l} \sqrt{k_{s g s}^{3}} / \bar{\Delta}+\left\langle\dot{S}_{k s g s}^{s p}\right\rangle_{l} \\
& +\frac{\partial}{\partial x_{i}}\left[\tau_{i j}^{s g s}\left\langle u_{i}\right\rangle_{L}+\langle\rho\rangle_{l}\left(v+c_{k} \bar{\Delta} \sqrt{k_{s g s}}\right) \frac{\partial k_{s g s}}{\partial x_{j}}\right. \\
& \left.+\langle\rho\rangle_{l} \frac{v_{t}}{\operatorname{Pr}_{t}}\langle R\rangle_{L} \frac{\partial\langle T\rangle_{L}}{\partial x_{j}}\right] .
\end{aligned}
$$

In Eq. (5), the model coefficients for eddy viscosity, $c_{v}$, and SGS transport of $k_{s g s}, c_{k}$ are computed dynamically and the dissipation coefficient, $c_{\epsilon}$ is assumed to be constant and unity (Irannejad and Jaberi, 2014). The subgrid stress $\tau_{i j}^{s g s}$ is assumed to be proportional to the eddy viscosity as

$\tau_{i j}^{s g s}=-2\langle\rho\rangle_{l} v_{t}\left(\left\langle S_{i j}\right\rangle_{L}-\frac{1}{3}\left\langle S_{k k}\right\rangle_{L} \delta_{i j}\right)+\frac{2}{3}\langle\rho\rangle_{l} k_{s g s} \delta_{i j}$

The SGS velocity correlations in the energy and scalar equations are also modeled with similar gradient type closures:

$H_{i}^{\mathrm{sgs}}=-\langle\rho\rangle_{l} \frac{v_{t}}{\operatorname{Pr}_{t}} \frac{\partial\langle H\rangle_{L}}{\partial x_{i}}$,

$J_{\alpha i}^{s g s}=-\langle\rho\rangle_{l} \frac{v_{t}}{S c_{t}} \frac{\partial\left\langle\phi_{\alpha}\right\rangle_{L}}{\partial x_{i}}$,

where $\langle H\rangle_{L}=\langle E\rangle_{L}+\langle p\rangle_{l} /\langle\rho\rangle_{l}$ is the total filtered enthalpy, and $\operatorname{Pr}_{t}$ and $S c_{t}$ are turbulent Prandtl and Schmidt numbers, respectively, which are assumed to be 0.5 .

\section{Lagrangian droplet equations}

The droplet motion is simulated using Basset-Bousinesq-Oseen (BBO) equation (Crowe et al., 1998; Sirignano, 2010; Loth, 2000) for gas-droplet systems. Since the droplet density is much larger than the career fluid density and the droplet size and number density in the major portion of the spray is small, the droplet collisions are neglected. It was shown by Irannejad and Jaberi (2014) that for the single hole evaporating sprays of the kind considered in this work, the effect of droplet collisions on the spray behavior is indeed negligible. The Basset force, the added mass term and the Saffman lift force (which are known to be small compared to the drag force) are also neglected in our simulations. Under these assumptions, the Lagrangian equations describing the droplet or particle (identified by subscript $p$ ) motion are:

$\frac{d \boldsymbol{x}_{p}}{d t}=\boldsymbol{u}_{p}$,

$\frac{d \boldsymbol{u}_{p}}{d t}=\frac{\boldsymbol{u}_{r e l}}{\tau_{p}}$.

In Eq. (10), the relative velocity is $\boldsymbol{u}_{r e l}=\boldsymbol{u}_{g}-\boldsymbol{u}_{p}$, where the gas velocity at the droplet location, $\boldsymbol{u}_{g}=\langle\boldsymbol{u}\rangle_{L}+\boldsymbol{u}^{*}$, is reconstructed 
using the filtered velocity and a SGS velocity and the subgrid velocity $u_{s g s}=\sqrt{2 k_{s g s} / 3}$, computed by a stochastic subgrid velocity model (Pozorski and Apte, 2009):

$d u_{i}^{*}=-\frac{u_{i}^{*}}{\tau_{L}^{*}} d t+\sqrt{\frac{2 u_{s g s}^{2}}{\tau_{L}^{*}}} d W_{i}$

where $d W_{i}$ is an increment of the Wiener process (Gardiner et al., 1990) and $\tau_{L}^{*}$ is based on the SGS time scale and the ratio of SGS velocity to the filtered relative velocity. This velocity reconstruction is intended to cover a wide range of droplet Stokes numbers (Pozorski and Apte, 2009). Furthermore, this relative velocity is modified by the wake effects of nearby droplets in the spray (Irannejad and Jaberi, 2014). Looking for nearby droplets of a specific or concerned droplet, possible upstream droplets with the lowest relative angle, minimum distance and maximum relative size are considered. The correction factor to the droplet relative velocity is computed from the fraction of the concerned droplet relative velocity in the wake of leading droplets. In this procedure, the relative position and size of the upstream droplets with respect to the concerned droplet are taken into account. This modified relative velocity is then used for calculating the droplet motion, heat and mass transfers.

The droplet or particle response time, $\tau_{p}=\tau_{s t} / f_{d}\left(R e_{s l}\right)$ is a function of droplet Stokes time, $\tau_{s t}=d_{p}^{2} \rho_{l} / 18 \mu_{g}$ and the drag factor, $f_{d}\left(R e_{s l}\right)$, which is the correction to the Stokes drag as a function of Reynolds number, $R e_{s l}=\rho_{g} d_{p} u_{\text {rel }} / \mu_{g}$. At low Reynolds numbers $\left(R e_{s l}<100\right)$, the drag factor includes the effect of the evaporation as a function of blowing Reynolds number, $R e_{b w}$ (Bellan and Harstad, 1987). For higher Reynolds numbers $\left(100<R e_{s l}<10^{5}\right)$, the evaporation effect on the drag is neglected and the standard drag factor for a sphere is used (Crowe et al., 1998). The drag factor is modified for the distorting drop, using the simple assumption that the drag coefficient of a distorting drop should lie between the lower limit of a rigid sphere and the upper limit of a disk, with the distortion computed from the TAB model (Liu et al., 1993).

\section{Spray breakup model}

To simulate the spray, the liquid jet is modeled with a commonly used "blob" model (Gorokhovski and Herrmann, 2008; Jiang et al., 2010; Reitz, 1987). In this model, the initial development of the liquid jet is modeled by a set of round liquid blobs injected from the nozzle with a similar mass flow rate. These large drops then go through a series of breakup processes mimicing the primary and secondary breakups. This is based on the reasonable assumption that the atomization and fragmentation of the liquid jet, blobs or drops are indistinguishable processes within the dense liquid core region near the injector nozzle exit. As a result of the simplification of the liquid jet model, the injected blob properties rely on empirical models and coefficients to keep the injection mass flow rate similar to that of the experiment. The coefficients used in the model are listed in Table 2. The initial size of blobs is found from the nozzle diameter and the experimentally measured area-contraction coefficient which varies according to the injection pressure for different nozzles (Kastengren et al., 2012; Pickett, 2013). The discharge coefficient depends on the nozzle and varies between 0.8 and 0.84 for nozzle diameters of 100 and $498 \mu \mathrm{m}$. The initial

Table 2

Model coefficients for the blob injection.

\begin{tabular}{lll}
\hline Coefficient & Model & Values \\
\hline$c_{u}$ & Velocity coefficient of blob injection & Measured, 0.7 to 1.0 \\
$c_{n o z}$ & Blob injection spreading angle & Measured, 0.26 to 0.276 \\
$c_{d}$ & Nozzle discharge coefficient & Measured, 0.8 to 0.84 \\
\hline
\end{tabular}

blob velocity is calculated from the Bernoulli equation with a correction coefficient as $u_{p, i n j}=c_{u} \sqrt{2 p_{i n j} / \rho_{l}}$. The experimentally found coefficient, $c_{u}$ is the product of the discharge and the area contraction coefficients and varies from 0.7 to 1.0 (Kastengren et al., 2012; Pickett, 2013). The spray spreading angle with which the blobs are injected is also found from an experimental correlation (Siebers et al., 1999) as $\tan \left(\theta_{s p} / 2\right)=c_{n o z}\left[\left(\frac{\rho_{g}}{\rho_{l}}\right)^{0.19}-0.0043 \sqrt{\frac{\rho_{l}}{\rho_{g}}}\right]$, where $c_{n o z}$ depends on the nozzle diameter according to the experiment (varying from 0.255 for the nozzle diameter of $100 \mu \mathrm{m}$ to 0.276 for the diameter of $0.498 \mu \mathrm{m}$ ). For the blob and droplet breakup, a stochastic breakup model capable of generating a broad range of droplet sizes at high Weber numbers is employed in which the characteristic radius of droplets is assumed to be a time-dependent stochastic variable with a given initial distribution (Kolmogorov, 1941; Gorokhovski and Saveliev, 2003). The breakup of parent drops into daughter droplets is viewed as the temporal and spatial evolution of the distribution function according to the following Fokker-Planck (FP) differential equation.

$\frac{\partial \mathfrak{I}\left(\chi, t^{*}\right)}{\partial t}=-\frac{\langle\varsigma\rangle}{\tau_{b u}} \frac{\partial \mathfrak{I}\left(\chi, t^{*}\right)}{\partial \chi}+\frac{1}{2} \frac{\left\langle\varsigma^{2}\right\rangle}{\tau_{b u}} \frac{\partial^{2} \mathfrak{I}\left(\chi, t^{*}\right)}{\partial \chi^{2}}$,

where $t^{*}$ is the scaled time based on the breakup time $\tau_{b u}$. In Eq. (12), $\mathfrak{I}\left(\chi, t^{*}\right)$ is the distribution function for $\chi=\ln \left(r / r_{0}\right)$, the logarithm of droplet size scaled by the parent droplet radius $r_{0}$, and $\langle\varsigma\rangle$ and $\left\langle\varsigma^{2}\right\rangle$ are the first two moments of the fragmentation intensity spectrum, assumed to be Gaussian. The two moments are set to be $\langle\varsigma\rangle=-0.36$ and $\left\langle\varsigma^{2}\right\rangle=0.14$ (Gorokhovski and Saveliev, 2008). The breakup frequency, $\tau_{b u}=\sqrt{3} \sqrt{\frac{\rho_{l}}{\rho_{g}}} \frac{r_{0}}{u_{r_{l} l_{b u}}}$, is tied to the instabilities on the surface of the droplet (O'Rourke et al., 1987). These instabilities are assumed to be induced by the relative velocity fluctuation of the droplet with respect to the gas, $u_{r e l_{b u}}$, which is estimated based on the Lagrangian velocity fluctuations for the droplet (Irannejad and Jaberi, 2014). With $\tau_{\text {age }}$ being the total time of droplet exposure to the gas, $u_{r e l_{b u}}$ is assumed to be equal to the Lagrangian root mean square (r.m.s.) of the relative velocities as:

$u_{r e l_{b u}}=\sqrt{\frac{\left|\boldsymbol{u}_{r e l}-\left\langle\boldsymbol{u}_{r e l}\right\rangle_{t}\right|^{2} \Delta t}{\tau_{a g e}}}$,

where $\left\langle\mathbf{u}_{r e l}\right\rangle_{t}$ is the averaged relative velocity of the droplet within time period of $\tau_{\text {age }}$.

The breakup proceeds for the new born droplets till they reach a stable or critical radius. The critical radius can be obtained by a balance between the disruptive hydrodynamic and capillary forces according to a critical Weber number (Plich and Erdman, 1987; Gelfand, 1996).

\section{Droplet heat and mass transfer models}

With the assumptions of Fourier heat conduction and Fickian mass diffusion, the following spherically symmetric, one dimensional, multicomponent unsteady continuity, energy and species equations (Torres et al., 2003; Srivastava et al., 2013; Irannejad and Jaberi, 2014) inside each individual droplet are solved.

$$
\begin{aligned}
& \frac{\partial \rho_{l}}{\partial t}+\frac{1}{r^{2}} \frac{\partial}{\partial r}\left(r^{2} \rho_{l} v_{l}\right)=0, \\
& \frac{\partial\left(\rho_{l} \phi_{l \alpha}\right)}{\partial t}+\frac{1}{r^{2}} \frac{\partial}{\partial r}\left(r^{2} \rho_{l} v_{l} \phi_{l \alpha}\right)=\frac{1}{r^{2}} \frac{\partial}{\partial r}\left(r^{2} \rho_{l} D_{l} \frac{\partial \phi_{l \alpha}}{\partial r}\right), \\
& \frac{\partial\left(\rho_{l} T_{l}\right)}{\partial t}+\frac{1}{r^{2}} \frac{\partial}{\partial r}\left(r^{2} \rho_{l} v_{l} T_{l}\right)=\frac{1}{c_{p} r^{2}} \frac{\partial}{\partial r}\left(r^{2} \lambda_{l} \frac{\partial T_{l}}{\partial r}\right) .
\end{aligned}
$$

The mass and energy conservations provide necessary droplet interface quantities like the radial velocity, $v_{l s}$, and the species mass fraction and temperature gradients at the droplet surface. Moreover, 
global droplet mass and energy quantities such as the total change in the droplet internal energy (i.e. $d(m e)_{p} / d t$ ) are also obtained by integrating Eqs. (14)-(16). The internal droplet circulation, caused by the relative motion of gas and liquid, and its effect on the droplet variables are accounted for by changing the effective liquid mass and thermal diffusivities (Abramzon and Sirignano, 1989).

For a multicomponent droplet, the mass flux of species $\alpha$ at the droplet interface may be written as

$\dot{m}_{\alpha}=\phi_{l s \alpha} 2 \pi r_{s} \rho_{g s} D_{g \alpha} S h_{\alpha} \ln \left(1+B_{\mathrm{M} \alpha}\right)$,

where the Spalding mass transfer number, defined as,

$B_{M \alpha}=\frac{\phi_{g s \alpha}-\phi_{\infty \alpha \alpha}}{\phi_{I s \alpha}-\phi_{g s \alpha}}$,

relates the liquid and gas interface mass fractions $\left(\phi_{l s x}\right.$ and $\left.\phi_{g s \alpha}\right)$ to the free stream (with respect to the droplet) mass fraction $\phi_{\infty \alpha}$. The species mass diffusivity $D_{g \alpha}$ is found from the species Schmidt number $S c_{\alpha}$ using the viscosity and density of the gas mixture. For the heat and mass transfers between the droplet and surrounding gas, the following well-known Ranz-Marshall correlations for the Sherwood and Nusselt numbers are used based on the droplet Reynolds number $R e_{s l}$, the Prandtl number and the Schmidt number (Ranz and Marshall, 1952).

$N u^{*}=2+0.552 \operatorname{Re}_{s l}^{1 / 2} \operatorname{Pr}^{1 / 3} ; \quad \operatorname{Sh}_{\alpha}^{*}=2+0.552 \operatorname{Resl}^{1 / 2} \operatorname{Sc}_{\alpha}^{1 / 3}$

The Prandtl and Schmidt numbers are assumed to be the same and equal to 0.7 . To account for the surface blowing effect of the evaporated liquid, Sherwood and Nusselt numbers are modified based on the film model of Abramzon and Sirignano (1989). The overall mass flow rate at the droplet surface is

$\dot{m}_{p}=4 \pi r_{s}^{2} \rho_{l s}\left(\dot{r}_{s}-v_{l s}\right)$,

where the surface regression rate is found by summing over all species as:

$\left(\dot{r}_{s}-v_{l s}\right)=\frac{\rho_{g s} \sum_{\alpha} \phi_{l s \alpha} D_{g \alpha} S h_{\alpha} \ln \left(1+B_{M \alpha}\right)}{2 r_{s} \rho_{l s}}$.

Finally, the interphase coupling effects are represented by the conservation of mass for species $\alpha$ and energy at the droplet interface as:

$$
\begin{aligned}
& 2 r_{s} \rho_{l s}\left(v_{l s}-\dot{r}_{s}\right)\left(\phi_{g s \alpha}-\phi_{l s \alpha}\right)+\left.2 r_{s} \rho_{l s} D_{l s} \frac{\partial \phi_{\alpha}}{\partial r}\right|_{l s} \\
& \quad-\rho_{g s} D_{g \alpha} S h_{\alpha} \ln \left(1+B_{M \alpha}\right)=0,
\end{aligned}
$$

$$
\begin{aligned}
& 2 r_{s} \sum_{i} L_{v \alpha} \rho_{l s}\left[\left(\dot{r}_{s}-v_{l s}\right) \phi_{l s \alpha}+\left.D_{l \alpha} \frac{\partial \phi_{\alpha}}{\partial r}\right|_{l s}\right]-\left.2 r_{s} \lambda_{l} \frac{\partial T}{\partial r}\right|_{l s} \\
& \quad+\lambda_{g} N u\left(T_{g \infty}-T_{s}\right)=0 .
\end{aligned}
$$

\section{Spray source terms}

The effects of droplets on the carrier gas are included via a series of source/sink terms in the gas continuity, momentum, energy and scalar equations (Eqs. (1)-(4)) and subgrid kinetic energy equation (Eq. (5)). These terms are evaluated by volumetric weighted averaging and interpolation of the Lagrangian variables as (Irannejad and Jaberi, 2014):

$$
\left\langle\dot{S}_{\text {Mass }}^{s p}\right\rangle_{l}=-\sum_{p} \frac{w_{p}}{\Delta V} \dot{m}_{p},
$$

$$
\left\langle\dot{S}_{m i}^{s p}\right\rangle_{l}=-\sum_{p} \frac{w_{p}}{\Delta V}\left[m_{p} \frac{d u_{p i}}{d t}+\dot{m}_{p} u_{p i}\right],
$$

$\left\langle\dot{S}_{E}^{s p}\right\rangle_{l}=-\sum_{p} \frac{w_{p}}{\Delta V}\left[\frac{d(m e)_{p}}{d t}+\frac{1}{2} \dot{m}_{p} \boldsymbol{u}_{p}^{2}+m_{p} \boldsymbol{u}_{p} \cdot \frac{d \boldsymbol{u}_{p}}{d t}\right]$,

$$
\begin{aligned}
\left\langle\dot{S}_{\alpha}^{s p}\right\rangle_{l}= & -\sum_{p} \frac{w_{p}}{\Delta V} \dot{m}_{\alpha}, \\
\left\langle\dot{S}_{\text {ksgs }}^{s p}\right\rangle_{l}= & \left(\left\langle\dot{S}_{m i}^{s p}\left\langle u_{i}\right\rangle_{L}\right\rangle_{l}-\left\langle\dot{S}_{m i}^{s p}\right\rangle_{l}\left\langle u_{i}\right\rangle_{L}\right) \\
& -\left(\left\langle\dot{S}_{\text {Mass }}^{s p} k_{\text {total }}\right\rangle_{l}-\left\langle\dot{S}_{\text {Mass }}^{s p}\right\rangle_{l} \frac{\left\langle u_{i}\right\rangle_{L}\left\langle u_{i}\right\rangle_{L}}{2}\right) .
\end{aligned}
$$

\section{Results and discussions}

The numerical simulations are conducted for conditions similar to those considered in experiments performed at Sandia National Laboratory as a part of engine combustion network (ECN) (Pickett, 2013; Siebers et al., 1999; Siebers et al., 1998; Pickett et al., 2010; Bardi et al., 2012). In these experiments, the fuel spray behavior in a closed combustion chamber is studied. The combustion chamber, as shown in Fig. 2 is approximated to be like a $105 \mathrm{~mm}$ cube. The fuel injector is located in one side of the chamber, and the nozzle diameter ranges from 0.1 to $0.5 \mathrm{~mm}$ with the fuel injection pressure varying between 50 and $200 \mathrm{MPa}$. The liquid injection pressure refers to the difference between liquid pressure inside the injector and the gas pressure in the chamber. The liquid pressure is much higher than the gas pressure in the chamber, leading to the liquid jet to deeply penetrate and drive the gas with very high velocities. The temperature and density of the gas in the chamber (which is mostly nitrogen) is varied between 700 and $1300 \mathrm{~K}$ and 3 and $60 \mathrm{~kg} / \mathrm{m}^{3}$, respectively. In this work, the effects of initial gas temperature and density, the injector pressure and nozzle diameter on the spray are studied and compared with the experiment. We will also compare the LES predicted global spray quantities like the liquid and vapor penetration lengths as well as the fuel vapor mass fraction profiles with the experiment. The liquid penetration length is the maximum extent of liquid-phase penetration during the injection. As recommended by ECN (Pickett, 2013), the liquid penetration length is defined here to be the axial location at which the liquid volume fraction drops to $0.15 \%$. The vapor penetration length is defined to be the maximum distance in which the fuel mass fraction reaches to $0.1 \%$.

The liquid and gas phase parameters are set according to experimental conditions. The initial diameter of liquid blobs and their injection velocities are calculated based on the nozzle diameter and the injection pressure according to the spray mass flow rate.

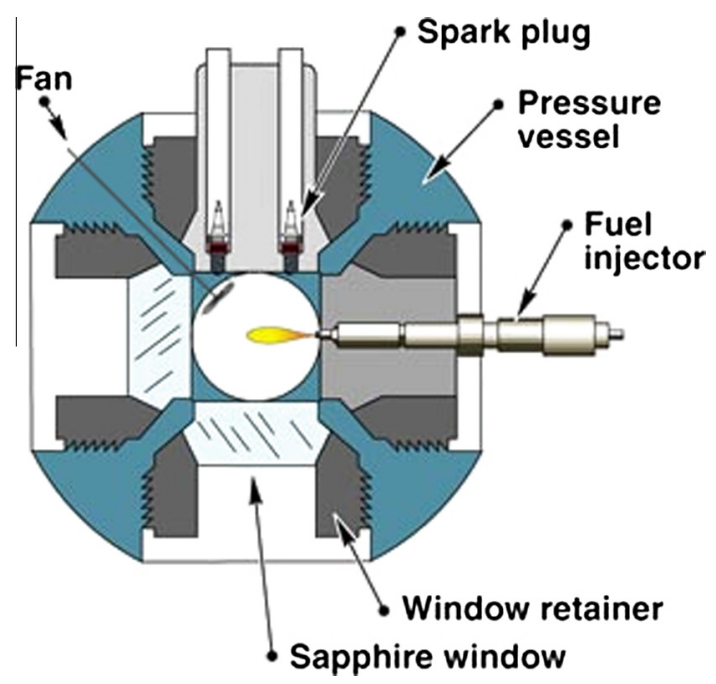

Fig. 2. Schematic of Sandia's closed chamber spray apparatus (Pickett, 2013). 
Depending on the liquid injection pressure, the liquid injection velocity ranges between 400 and $700 \mathrm{~m} / \mathrm{s}$. The maximum liquid Mach number is then always below 0.5. Additionally, the maximum induced gas velocity is observed to be less than half of the injection velocity. Since the chamber temperature is high, the gas flow in the chamber is always subsonic (the maximum flow Mach number is around 0.6). The injected fuel properties are obtained and computed from the data and correlations given by Poling et al. (2001). A rectangular numerical domain is used for LES with a length of $105 \mathrm{~mm}$ in the axial direction. The chamber has a somehow irregular shape in lateral directions. While the exact geometry of the side walls is irrelevant to the spray flow, the total mass of the gas in the chamber should match the experimental conditions for larger nozzles. This can be important for large injector nozzles for which gas entertainment is higher. Here, the length of the chamber in lateral directions is considered to be $100 \mathrm{~mm}$. On the other hand, preliminary simulations showed that the induced gas flow in the chamber with the smaller nozzle of $100 \mu \mathrm{m}$ is limited to the spray region and simulations with the entire chamber and with only half of the chamber size (i.e. $50 \mathrm{~mm}$ ) around the spray in lateral directions yielded similar results. Therefore, for this nozzle, only half of the chamber size in lateral directions is simulated with free-stream boundary conditions at lateral boundaries. The grid resolution for different nozzles is chosen to be twice of the nozzle diameter. Based on our LES study (Irannejad and Jaberi, 2014) and as demonstrated further in this paper, this choice of grid resolution is sufficient for capturing the essential features of the flow and the turbulence in the gas.

To assess the importance of various incorporated spray submodels, a detailed study of them have been conducted in our previous paper (Irannejad and Jaberi, 2014). It was shown that a good comparison with experimental data for a wide range of spray and gas operating conditions is only possible when all processes involved in the spray and gas flow evolution are properly modeled. Some of the most important processes in the simulated spray and flow are the droplet wake interactions, turbulent kinetic energy budget at subgrid level as obtained by $k_{s g s}$ equation, and the finite rate heat/mass transfer. Detailed study of spray/gas submodels in our previous paper (Irannejad and Jaberi, 2014) indicates that the predicted spray features could differ significantly from the experiment if one of the important submodels is removed or improperly modeled. For example, due to fast droplet heating, particularly at high temperatures (e.g. $T=1000 \mathrm{~K}$ ), the rate of heat and mass transfers between phases is so high that the droplet interior cannot adjust to variations in droplet surface conditions. Therefore, the models developed based on the assumption of uniform droplet temperature and species distribution can lead to a significant underprediction of evaporation. In the next several sections, the global and local behavior of the spray and particularly the gas flow generated by the spray under different conditions are studied with the LES/spray model and its submodels already developed and tested (Irannejad and Jaberi, 2014).

\section{Global spray behavior}

For n-dodecane and n-heptane sprays, designated as "Spray A" and "Spray H" by ECN (Bardi et al., 2012; Pickett, 2013), respectively, there are experimental data on transient liquid and vapor penetrations. On the other hand, the mean liquid penetration lengths are available for n-hexadecane spray, designated as Spray CET for a range of gas densities and temperatures as well as different nozzle sizes and injection pressures. The main parameters of these sprays are listed in Table 3 . The information concerning the grid resolution, $\Delta$, the total number of grid points, $N_{G}$, the average number of instantaneous computational droplets, $N_{P C}$, and the computational cost based on the number of computational proces-
Table 3

Parameters of different simulated sprays.

\begin{tabular}{llll}
\hline Parameter & Spray A & Spray H & Spray CET \\
\hline Fuel & n-dodecane & n-heptane & n-hexadecane \\
$T_{\text {fuel }}(\mathrm{K})$ & 363 & 363 & 436 \\
$d_{\text {noz }}(\mu \mathrm{m})$ & 90 & 100 & $100-498$ \\
$p_{\text {inj }}(\mathrm{MPa})$ & 150 & 150 & $55-172$ \\
$\Delta t_{\text {inj }}(\mathrm{ms})$ & 1.5 & 6.0 & $2.0-4.0$ \\
$T_{g}(\mathrm{~K})$ & 900 & 1000 & $750-1300$ \\
$\rho_{g}\left(\mathrm{~kg} / \mathrm{m}^{3}\right)$ & 22.8 & 14.8 & $3.60-58.8$ \\
$\% x_{\mathrm{N}_{2}}$ & 89.71 & 89.71 & 100.0 \\
$\% x_{\mathrm{CO}_{2}}$ & 6.52 & 6.52 & 0.0 \\
$\% x_{\mathrm{H}_{2} \mathrm{O}}$ & 3.77 & 3.77 & 0.0 \\
\hline
\end{tabular}

Table 4

Computational parameters for different spray simulations.

\begin{tabular}{lllll}
\hline$d_{\text {noz }}(\mathrm{mm})$ & $\Delta(\mathrm{mm})$ & $N_{G}$ & $N_{P C}$ & $N_{\text {core }}$ \\
\hline $0.900,0.100$ & 0.2 & $20,000,000$ & $50,000-100,000$ & 128 \\
$0.246,0.267$ & 0.5 & $3,500,000$ & $50,000-100,000$ & 32 \\
0.498 & 1.0 & $1,000,000$ & 50,000 & 16 \\
\hline
\end{tabular}
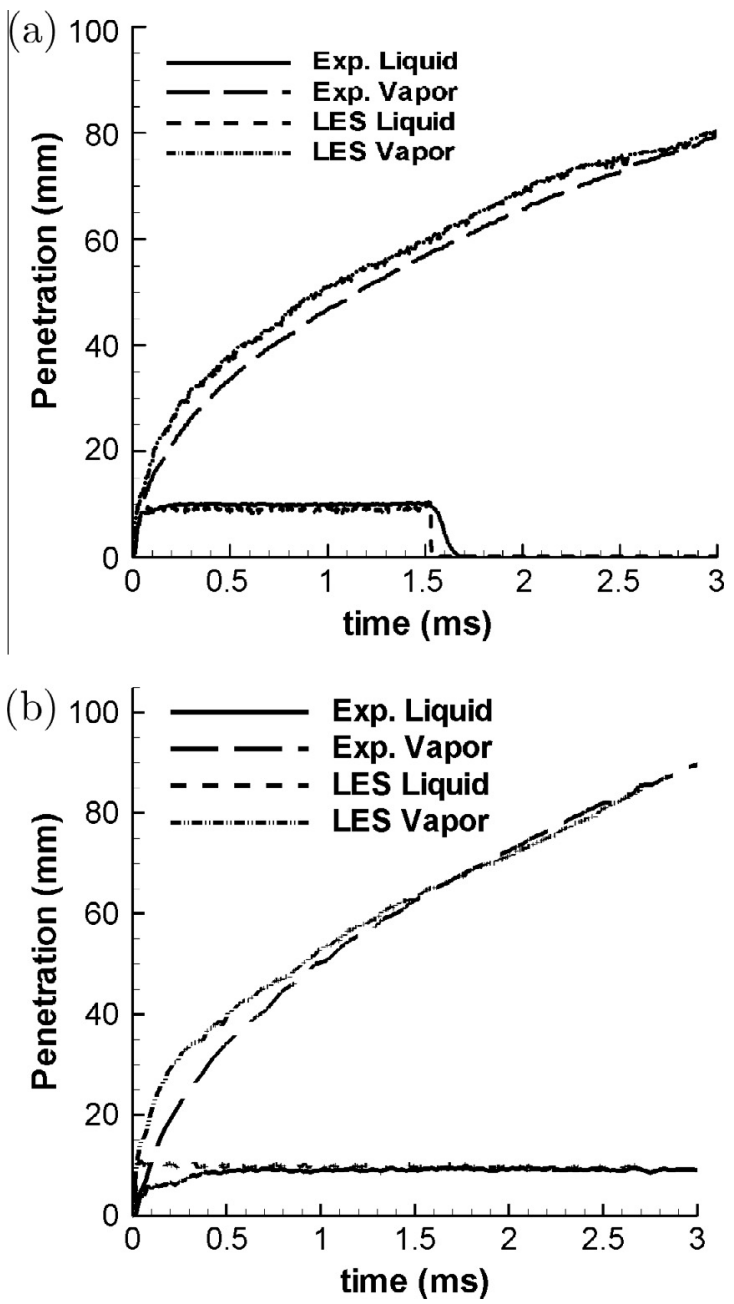

Fig. 3. Simulated and measured liquid and vapor penetration lengths versus time for different sprays. (a) Spray A, (b) Spray H.

sor cores, Ncore, for different injector nozzle diameters, $d_{n o z}$ are summarized in Table 4.

Fig. 3 shows the time variations of the liquid fuel spray and vapor phase penetration lengths for Sprays A and $\mathrm{H}$, obtained by 
the LES model and experiment. The results in Fig. 3 clearly indicate the capability of the two-phase LES to capture the global characteristics of the simulated sprays and the evaporated fuel in a highly unsteady and turbulent spray-induced gas flow. It is clear that the liquid spray quickly reaches a pseudo-steady condition while the vapor keeps on penetrating even after the liquid injection stops, hence making the spray flow essentially a transient phenomenon. The overprediction of liquid and vapor penetrations at early stages of the spray $\mathrm{H}$ development can be attributed to the details of liquid jet breakup and the effects that the jet breakup has on the evaporation of highly volatile n-heptane, when compared to ndodecane.

Fig. 4 compares the experimental values of mean liquid penetration length with the simulated results for spray CET as a function of density for two different gas temperatures. Here, the fuel is injected through a $0.246 \mathrm{~mm}$ diameter nozzle with the injection pressure of $138 \mathrm{MPa}$. The agreement of simulated results with the experimental data is fairly good for the sprays shown in Fig. 4 and all other sprays not shown in this paper (Irannejad and Jaberi, 2014). Once again, this indicates the accuracy and reliability of the LES model. As shown in Fig. 4, the penetration length decreases rapidly with increasing the gas density, more so at lower gas densities. Similarly, the liquid penetration length decreases with increasing the gas temperature for the range of gas densities considered in the experiment. The increase in gas density was shown to substantially increase the breakup of droplets and decrease the mean droplet size along the spray axis. The gas temperature through its effects on evaporation was shown to decrease the effect of gas flow dynamics when increased, making the numerical predictions of LES/spray less sensitive to the turbulence models at higher ambient gas temperatures (Irannejad and Jaberi, 2014).

\section{Local spray behavior}

To better understand the local spray behavior and to further validate the LES/spray submodels, available vapor mass fraction distributions for Spray H from ECN experiments are compared with LES results at different axial locations from the injector in Fig. 5. Evidently, the computed fuel mass fractions agree fairly well with the measured values. Fig. 5 also shows that the LES profiles are narrower and have higher central peak values in comparison to the experiment close to the nozzle. As explained below, this can be attributed to the spray source terms which are computed at finite difference (FD) grid points. Further downstream, the numerical

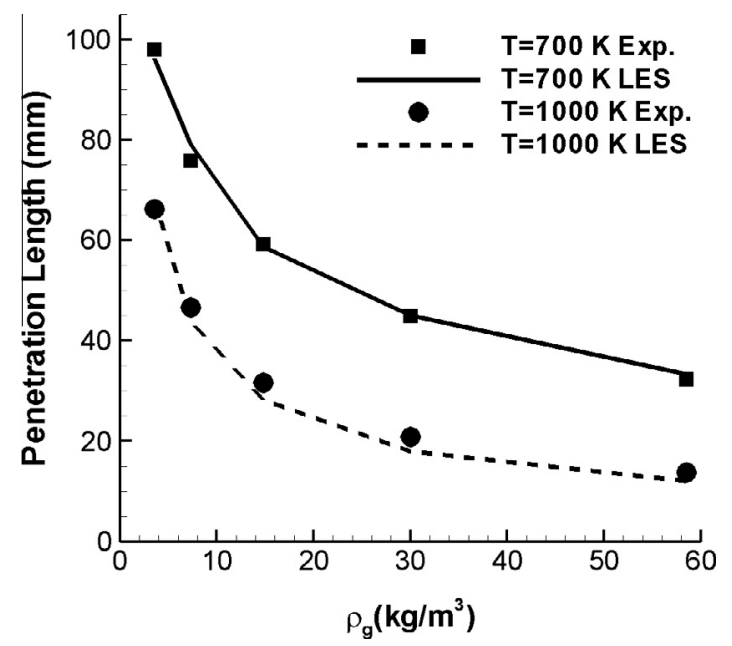

Fig. 4. Comparison of simulated and measured pseudo-steady liquid penetration lengths as a function of gas density at different temperatures in spray CET.
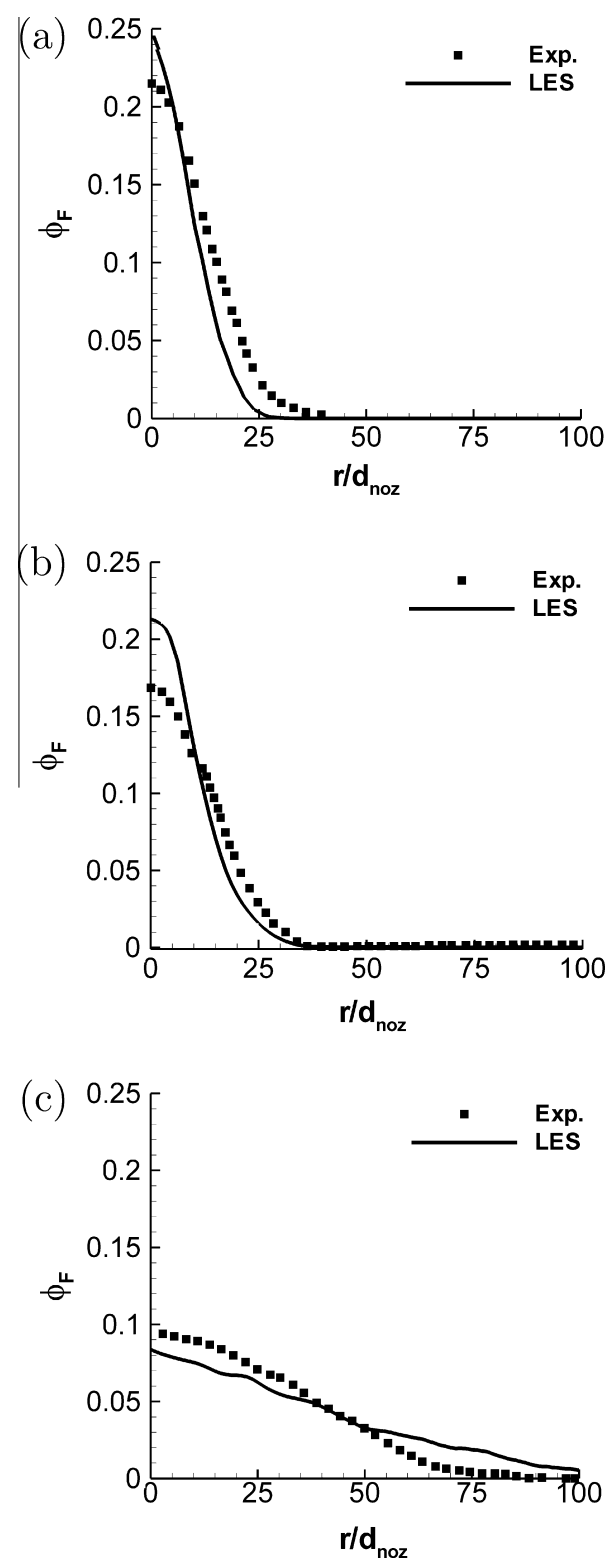

Fig. 5. Radial profiles of simulated and measured fuel mass fraction at different axial locations for Spray H. (a) $x=17 \mathrm{~mm}$, (b) $x=20 \mathrm{~mm}$, (c) $x=40 \mathrm{~mm}$.

values are slightly lower than the experimental values. This can be related to the upstream jet which becomes unstable faster and grows with a rate slightly more than that observed in the experiment.

Since most of experimental data are on spray CET and the rest of the paper deals with analysis of this spray, a detailed grid resolution study is also carried out for this spray. The grid study is conducted for radial profiles of azimuthally mean induced gas velocity and its resolved variance. Three grid resolutions of $3 d_{\text {noz }}, 2.5 d_{\text {noz }}$ and $2 d_{\text {noz }}$ are considered for the spray injected to the chamber with ambient gas temperature and density of $T=700 \mathrm{~K}$ and $\rho=14.8 \mathrm{~kg} / \mathrm{m}^{3}$. In the grid study of EulerianLagrangian models, one should consider the limitations of the "point particle in cell" assumption. A very fine LES grid leads to relatively high liquid volume fractions in the denser part of the spray which needs the reformulation of the carrier gas equations to include the finite liquid volume effect (Sirignano, 2005). This is not however the focus of this paper. The sensitivity of radial profiles of velocity and its variance to the grid size is shown in Fig. 6 
at an axial location of $20 d_{n o z} \sqrt{\rho_{l} / \rho_{g}}$. The mean velocity profiles in this figure become sharper and the variances increase with a decrease in LES grid size. Evidently, the mean profiles for the two finest grids are close to each other. However, the maximum velocities at the spray centerline for the two finest grids are still different. Expectedly, Fig. 6b shows that the velocity variance is more sensitive to the grid resolution than the mean velocity. However, for the two finest grids, the difference in peak values of the variance is less than $10 \%$. Therefore, it can be suggested that the finest considered grid can be used for the prediction of the low-order turbulence statistics. Similar behavior is observed for other nozzle sizes. It is to be noted that the highest number of droplets exist at the spray centerline. For a very fine grid, the local volume fraction at the spray centerline then may be so significant that cannot be neglected. On the other hand, the localized spray source terms at the centerline, which are computed at FD grid points with volumetric averaging of droplet quantities, become so large that the grid-based FD solver may become inaccurate. It may be safely stated that grid sizes finer than twice of the injector nozzle diameter cannot be used under the limitations of the present formulation.

In order to understand and explain the interactions between the spray with the gas flow generated by the spray, the conditional expectations of various flow variables are considered in Figs. 7-9 for the ambient gas temperature and density of $T=700 \mathrm{~K}$ and $\rho=14.8 \mathrm{~kg} / \mathrm{m}^{3}$. It is clear from Fig. 7a that the gas field has the highest density where the smallest droplets reside. This is due to evaporation of the "cold" heavy fuel which emanates mostly from smaller droplets. This is confirmed by the conditional fuel vapor mass fraction and gas temperature in Fig. $7 \mathrm{~b}$ and c. Corresponding to higher gas density and smaller droplets, there are higher fuel vapor mass fraction and more cooling of the gas. The conditional expected values of the total spray source term in the gas flow kinetic energy equation, $S_{k, t o t}^{s p}=\dot{S}_{m i}^{s p}\left\langle u_{i}\right\rangle_{L}-\dot{S}_{\text {Mass }}^{s p}\left\langle u_{i}\right\rangle_{L}\left\langle u_{i}\right\rangle_{L} / 2$, and the drag force contribution in this equation, $S_{k, d}^{s p}=-\frac{m_{p} d u_{p i} / d t}{\Delta V}\left\langle u_{i}\right\rangle_{L}$ are shown in Fig. 8. It is clear from the results in Fig. 8 that a major part of momentum transfer from droplets to the gas comes from the smallest droplets. It is also shown that for droplets larger than $50 \mu \mathrm{m}$, the evaporated vapor does not significantly contribute to the gas flow kinetic energy and the droplet drag force is the main driving force. On the other hand, for smaller droplets, the momentum is transferred to the gas mainly by the added vapor mass, as the difference between the total source term and the drag contribution increases rapidly with the decrease in droplet size. This indicates the significance of the evaporation and the fuel vapor in driving the gas flow. On the other hand, the conditional mean vorticity plot in Fig. 9, indicates that the maximum vorticity magnitudes occur at intermediate droplet diameters (around $50 \mu \mathrm{m}$ ). This suggests that the spray induces the highest perturbations in the gas when the droplet drag force and the vapor kinetic energy are both significant.

Fig. 10 shows the two-dimensional (2D) contours of the spray induced SGS and resolved kinetic energy, normalized by the square of the injection velocity for the intermediate gas density of $14.8 \mathrm{~kg} /$ $\mathrm{m}^{3}$ and relatively low gas temperature of $700 \mathrm{~K}$ at $t=2.0 \mathrm{~ms}$. For the spray injected into the quiescent chamber, the main source of generated gas flow is the spray. A gas jet is generated along the spray axis by the momentum transfered from the spray droplets. As explained below, depending on the spray parameters and chamber conditions, the spray generated gas jet remains stable down to an axial location comparable or longer than the liquid penetration length. On the other hand, the liquid-gas interface can directly create noticeable small-scale vorticity and turbulence in the gas phase (Li and Jaberi, 2009). However as Fig. 10 shows, this "small-scale" turbulence is expected to be rather weak
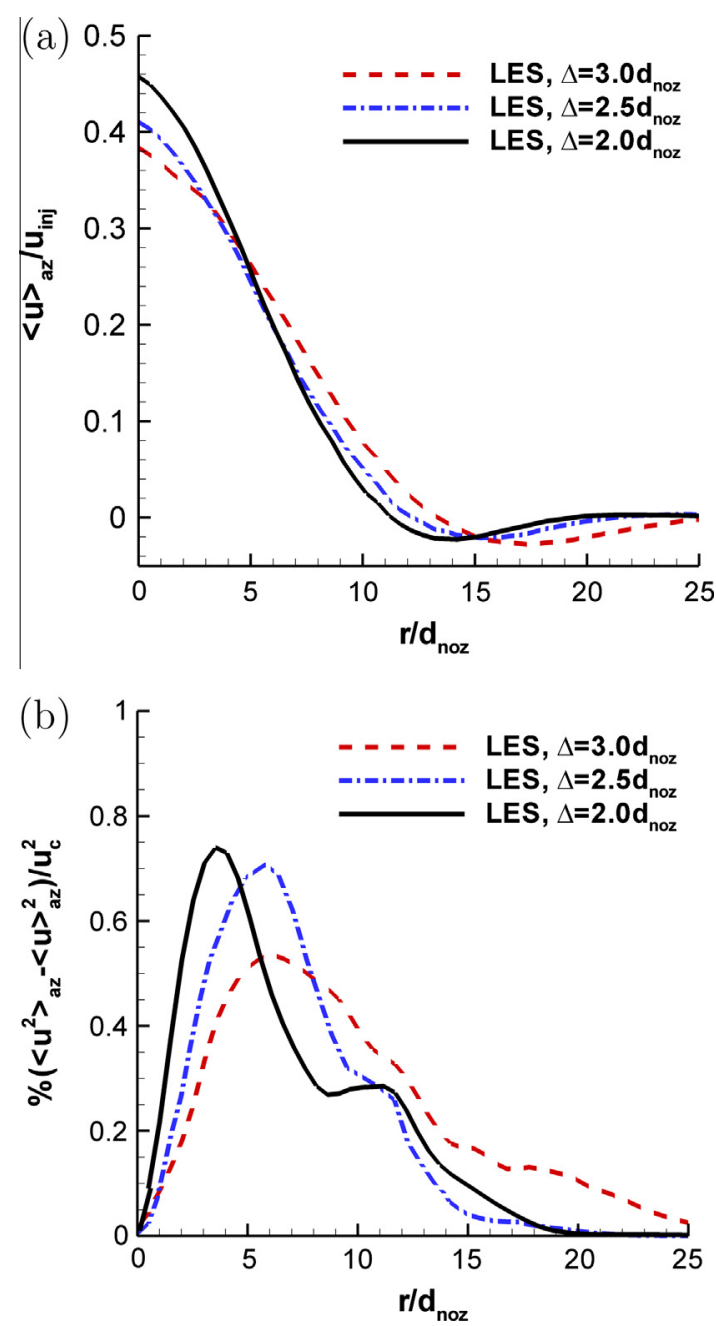

Fig. 6. Radial profiles of normalized induced gas velocity and its variance for different grid resolutions in Spray CET at non-dimensional axial distance of 20 from the injector. (a) Induced velocity, (b) induced velocity variance.

compared to the spray-inducted gas flow at resolved scales. Close to the spray axis, droplets tend to damp the small-scale turbulence because of their significant inertia. However, Fig. 10 shows that the small-scale turbulence is significant at the periphery of the spray in the near injector region. The small-scale turbulence enhances the poly-dispersity of droplets. The poly-dispersity of droplets in turn generates perturbations in the induced gas jet, making it more turbulent downstream.

The streamwise variation of instantaneous normalized spray source terms, integrated over cross-spray planes is shown in Fig. 11 for the ambient gas temperature and density of $700 \mathrm{~K}$ and $14.8 \mathrm{~kg} / \mathrm{m}^{3}$. The spray source terms are normalized with the injection velocity, $u_{i n j}$, nozzle diameter, $d_{n o z}$, ambient gas density, $\rho_{a}$ and internal energy, $e_{a}$ as

$$
\begin{aligned}
& \left(\frac{d S_{\text {Mass }}^{s p}}{d t}\right)_{n}=\frac{\dot{S}_{\text {Mass }}^{s p}}{\rho_{a} u_{i n j} / d_{n o z}}, \\
& \left(\frac{d S_{m x}^{s p}}{d t}\right)_{n}=\frac{\dot{S}_{m x}^{s p}}{\rho_{a} u_{i n j}^{2} / d_{n o z}}, \\
& \left(\frac{d S_{E}^{s p}}{d t}\right)_{n}=\frac{\dot{S}_{E}^{s p}}{\rho_{a} u_{i n j} e_{a} / d_{n o z}}, \\
& \left(\frac{d S_{k s g s}^{s p}}{d t}\right)_{n}=\frac{\dot{S}_{k s g s}^{s p}}{\rho_{a} u_{i n j}^{3} / d_{n o z}} .
\end{aligned}
$$



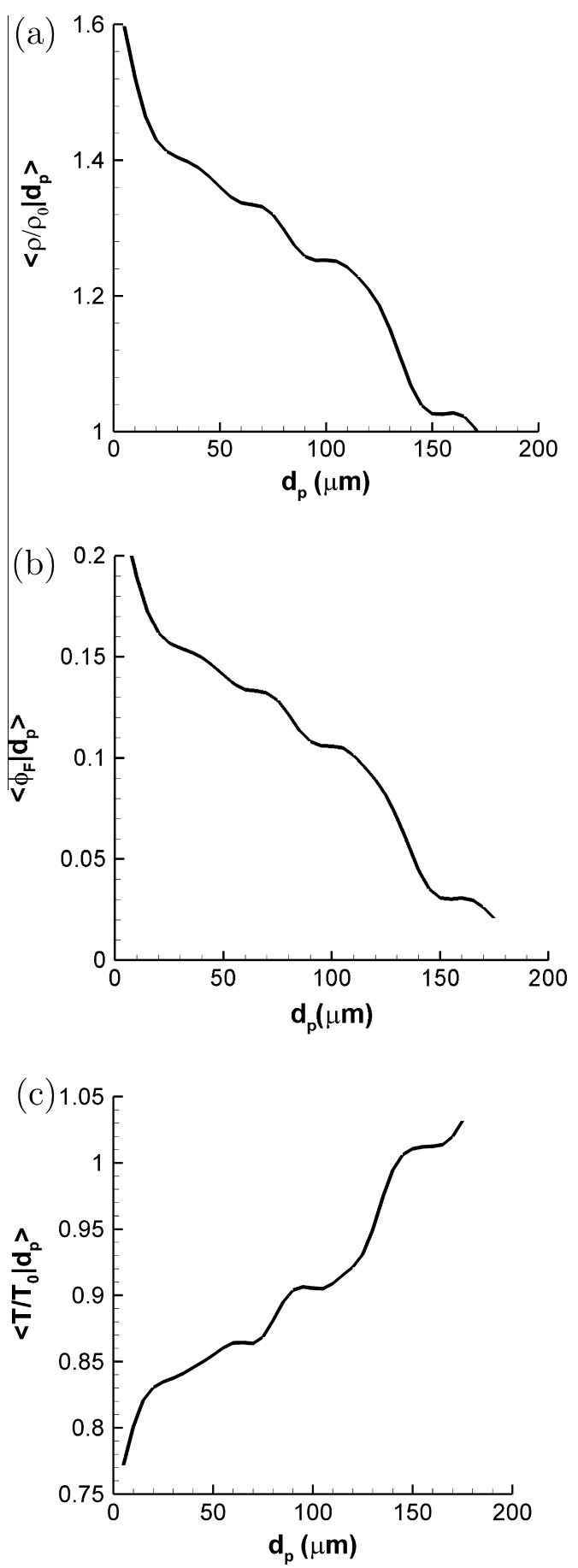

Fig. 7. Mean gas flow variables conditioned on the droplet diameter: (a) conditional normalized gas density, (b) conditional fuel vapor mass fraction, (c) conditional normalized gas temperature.

It is clear from the results in Fig. 11 that the spray source terms all increase in magnitude with the axial distance from the injector, reaching their peak values around $x=20 \mathrm{~mm}$ and then decrease. As the spray goes through breakup and smaller droplets are created in the near injector region, the interphase mass and energy transfers increases significantly. Further downstream, the evaporation and disappearance of droplets gradually reduce the spray effect on the gas. The spray mass source term is negligible very close to the injector because the evaporation from the large blobs, representing the liquid jet, is not significant. The spray mass source term is

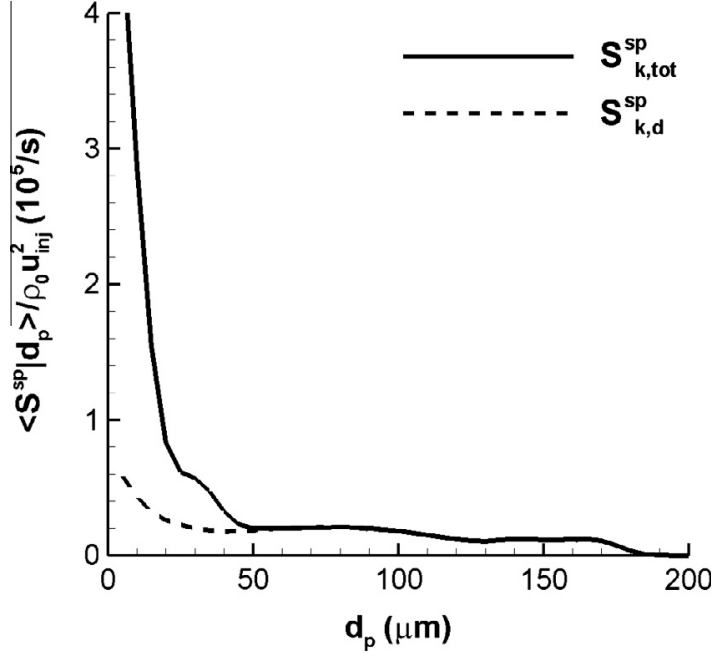

Fig. 8. Conditioned mean values of spray terms in the gas kinetic energy equation, conditioned on the droplet diameter.

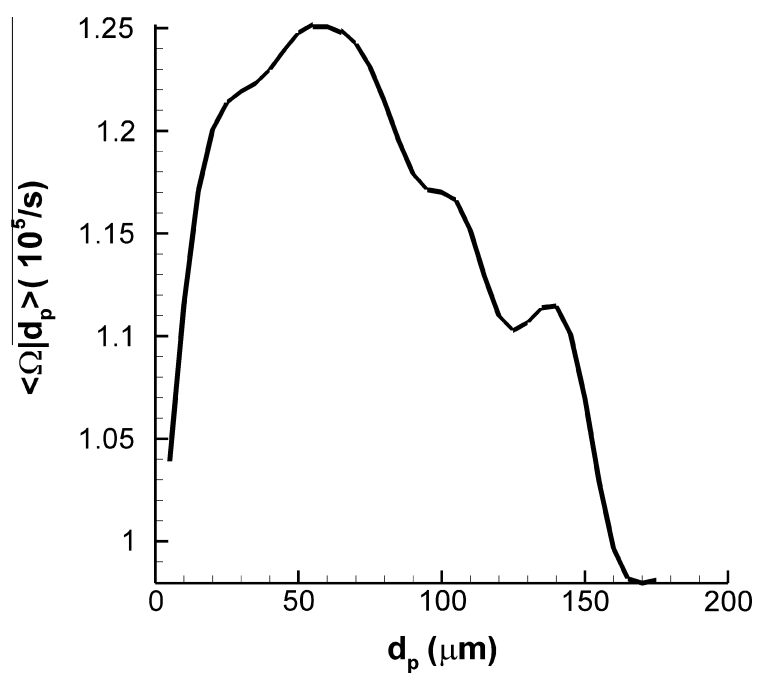

Fig. 9. Conditioned mean values of the gas flow vorticity, conditioned on the droplet diameter.

equivalent to the spray fuel vapor source term for the single component fuel considered here. In contrast to the mass source term, the momentum and energy source terms have finite values very close to the injector. As shown in Fig. 11, the subgrid kinetic energy source term is two orders of magnitude smaller than the spray source term to the filtered axial momentum equation. The liquid penetration length for the case considered in Fig. 11 is around $x=60 \mathrm{~mm}$. However, the spray source terms become negligible closer to the nozzle around $x=40 \mathrm{~mm}$. This indicates that the remaining atomized droplets at the tip of spray are not very significant in number and size as they are rapidly dispersed and disappear by the spray generated gas jet. As it is explained in more details in the next section, for the spray injected into relatively low ambient gas temperature of $700 \mathrm{~K}$, the vapor saturated gas jet does not have enough energy for significant and rapid evaporation of droplets. As a result, the evaporation is tied more to the gas jet turbulence. However, the spray source terms become insignificant at the tip of the spray, where the gas jet is highly turbulent and there is little interaction between droplets and gas turbulence. A source of turbulence in the gas jet is the perturbations in the spray variables as explained below. 


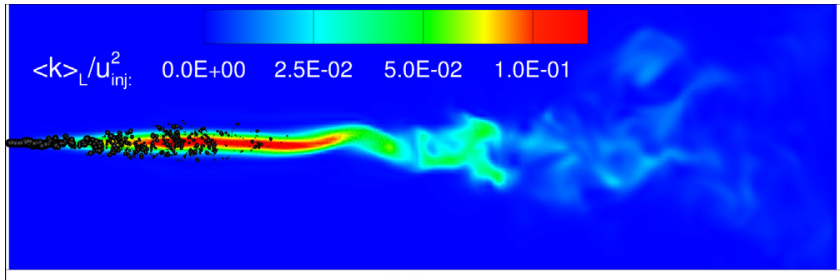

(a)

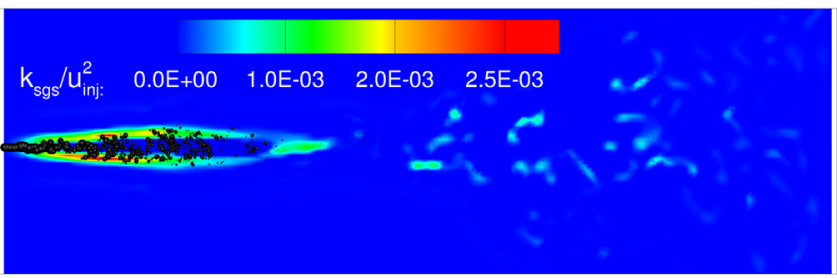

(b)

Fig. 10. Normalized resolved and subgrid gas kinetic energy for the gas density of $14.8 \mathrm{~kg} / \mathrm{m}^{3}$ and gas temperature of $700 \mathrm{~K}$ at $t=2.0 \mathrm{~ms}$. (a) resolved kinetic energy, (b) subgrid kinetic energy.
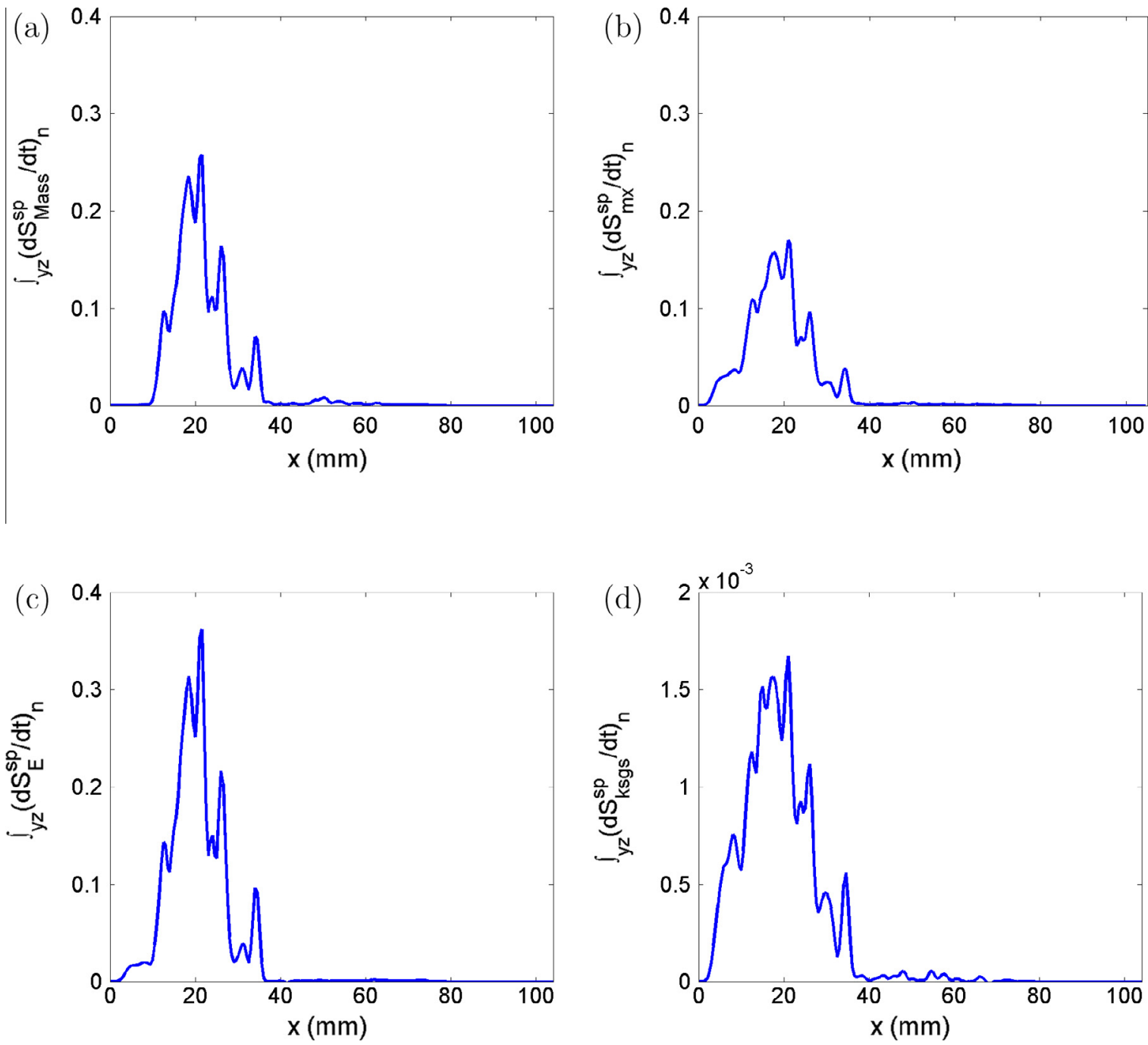

Fig. 11. Variation of spray source terms, integrated over the cross-spray planes versus axial distance from the injector. (a) Mass, (b) axial momentum, (c) total energy, (d) subgrid kinetic energy. 
Fig. 12 shows the iso-surfaces of normalized $Q$ i.e. $Q_{n}=\frac{Q}{\Omega_{i j} \Omega_{i j} / 2}=0.5$ for the spray induced gas flow at two chamber gas densities of 14.8 and $30.0 \mathrm{~kg} / \mathrm{m}^{3}$ and two gas temperatures of 700 and $1000 \mathrm{~K}$. The 3D structures in this figure show the very significant and complex turbulent flow generated by the spray and droplets in the gas. It is shown that the turbulent structures identified by $Q_{n}=0.5$, appear mostly away from the spray. It is also clear that more small scale turbulence is produced at lower chamber gas density after the breakdown of the spray induced gas jet. However, the turbulent structures appear in a shorter distance from the injector for the higher ambient gas density. The effect of ambient gas temperature on the turbulence is more complex. The flow generated by the spray produces more small scales at higher gas temperatures. As explained below, the liquid droplets induce larger perturbations in the induced gas jet at higher gas temperature of $1000 \mathrm{~K}$. As a result of stronger perturbations, the generated gas jet breaks into more small scales. These observations are confirmed by the integrated values of $Q_{n}$ in the chamber at different axial locations.

Fig. 13 shows the time variations of liquid penetration length for different initial chamber conditions. The maximum penetration point or the tip of the spray is where the droplet fuel evaporation rate in the spray equals the fuel mass reached to that point and as shown in Fig. 13 is fluctuating about a mean axial value in the simulated flow. Similar behavior is observed in the experiment (Siebers et al., 1998). The fluctuations in the spray length after it reaches to its steady state condition is negligible at higher ambient gas temperature of $1000 \mathrm{~K}$ but is very significant at lower gas temperature of $700 \mathrm{~K}$. At lower temperature of $700 \mathrm{~K}$ and at the tip of the spray, where there is a significant number of small droplets, the spray reaches to a nearly saturated, equilibrium condition with the entrained ambient gas after the early transient period. When such a saturated condition is reached, the only means for droplets to further vaporize is to penetrate more and reach the fresh gas. This is made possible by the spray generated turbulence as the pseudo-steady state (but highly oscillatory) part of the liquid penetration plot for lower gas temperature of $700 \mathrm{~K}$ in Fig. 13 shows. At lower temperature of $700 \mathrm{~K}$, a considerable number of small liquid droplets survive. These droplets interact rather significantly with the gas flow turbulence. At higher gas temperature of $1000 \mathrm{~K}$ the small droplets vaporize very rapidly in the much hotter gas.

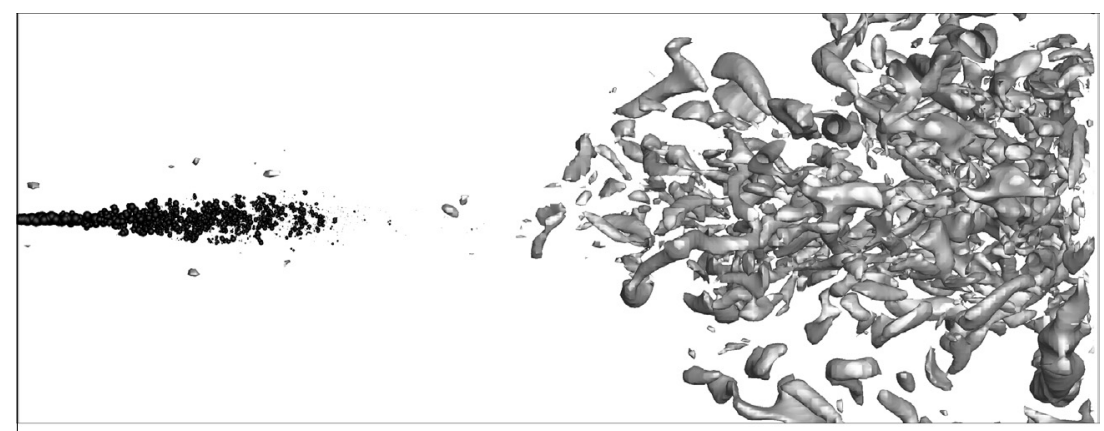

(a)

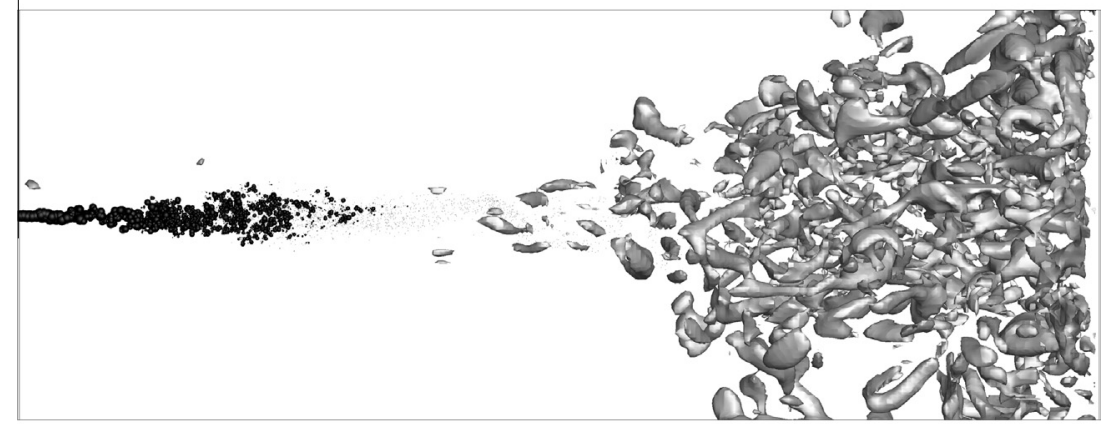

(b)

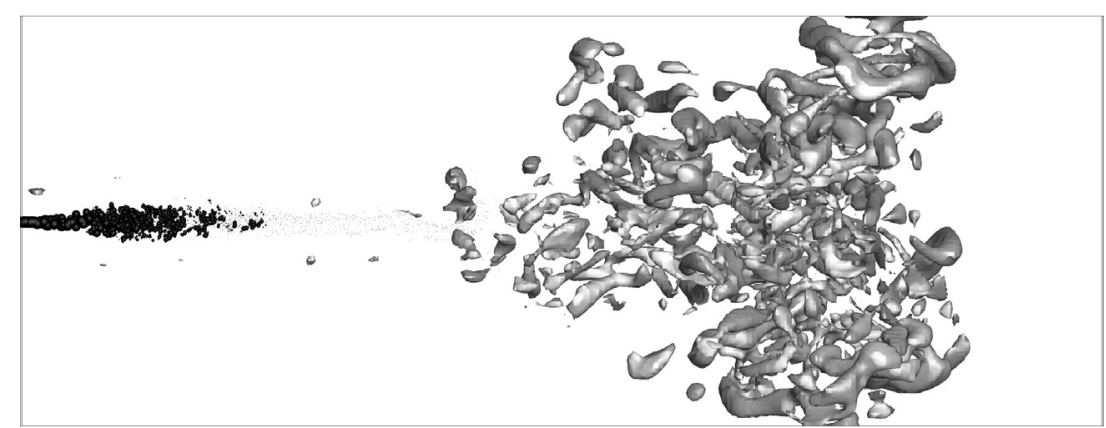

(c)

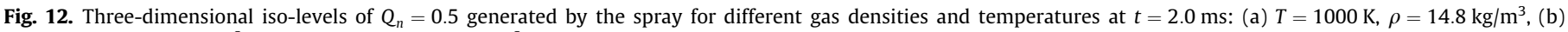
$T=700 \mathrm{~K}, \rho=14.8 \mathrm{~kg} / \mathrm{m}^{3}$, (c) $T=700 \mathrm{~K}, \rho=30.0 \mathrm{~kg} / \mathrm{m}^{3}$. 
Fig. 14 shows the Sauter mean diameter (SMD) of spray droplets for different gas densities and temperatures as a function of distance from the injector. One may note that the SMD is considerably larger (by a factor of 2) for the higher gas temperature at intermediate distances from the nozzle between $x=15$ and $30 \mathrm{~mm}$. This is due to rapid evaporation of the smaller droplets in the hot $(T=1000 \mathrm{~K})$ gas in this region of the flow. For the spray injected at lower gas temperature of $700 \mathrm{~K}$, the droplet SMD reaches to a constant value close to $1 \mu \mathrm{m}$ at long distances from the injector where fine droplets evaporate. This is due to the fact that a large number of fine droplets cannot evaporate any further in the cool vapor saturated induced gas jet. However as the gas jet goes through transition and breaks into smaller turbulent structures, the evaporated fuel is quickly convected and mixed, making it possible for the droplets to evaporate better in a less saturated environment. On the other hand, the SMD values along the spray axis are considerably lower (about three times) for the higher gas density case. This is due to more intense breakup of droplets.

Fig. 15 shows the 2D contours of gas temperature at different ambient (chamber) gas densities and temperatures. The oscillatory motion at the outer surface of the main gas jet near to the nozzle is shown to be enhanced and lead to smaller scale turbulent motions at downstream locations. The spray induced gas flow and turbulence efficiently disperse and mix the dense liquid and relatively cold evaporated high speed fuel with the low density, hot, quiescent surrounding gas. As large drops or blobs move in the gas, their high relative velocity creates significant dissipation which leads to heating of gas at the near injector region. As evident in Fig. 15, the gas temperature increase by this process is significant. The spray source terms in the energy equation are due to (1) the dissipation by droplet drag force (which is proportional to the droplet relative velocity), (2) the spray/droplet heating and (3) the evaporation. The spray/droplet heating and evaporation extract energy from the gas, while the dissipation increases the internal energy. In the near injector region, a dense pack of high speed large drops exists for which the heat and mass transfers are negligible. Therefore, the dissipation of gas kinetic energy to the internal energy is the most significant near the injector. Further downstream, the gas temperature in the spray region is significantly reduced by the cooling effect of evaporating droplets. It is noteworthy to mention that the major part of this cooling is not due to the latent heat of evaporation, but due to mixing of the relatively cold vaporized fuel around droplet surface with the higher temperature gas outside the droplet boundary layer. The low temperature regions of the

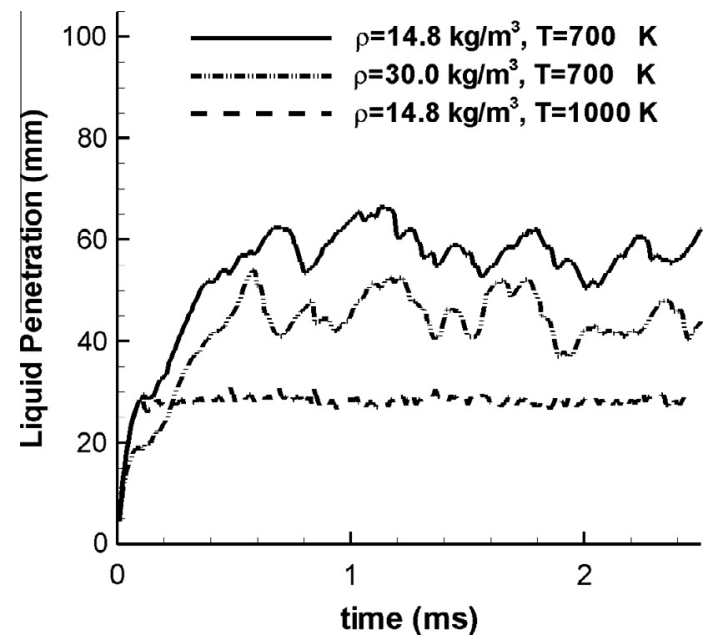

Fig. 13. Spray penetration as a function of time at different gas densities and temperatures.

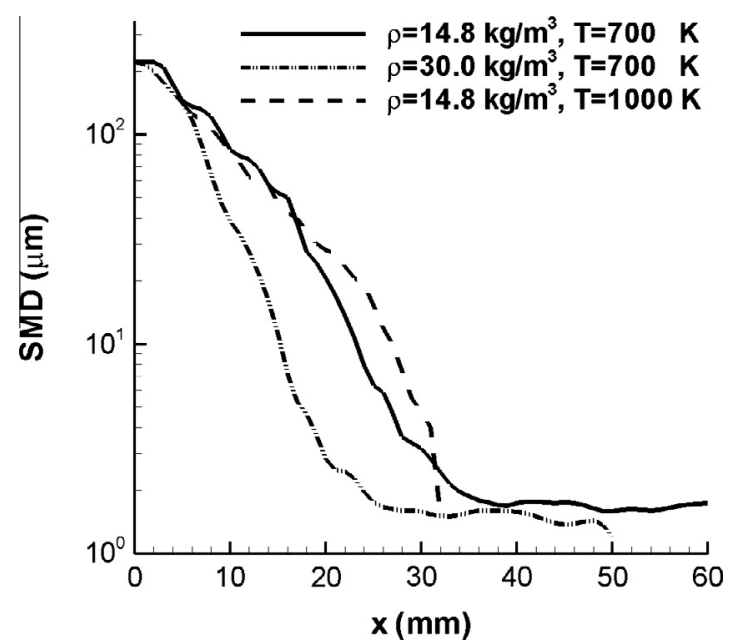

Fig. 14. Sauter mean diameter (SMD) of droplets as a function of distance from the injector at different gas densities and temperatures.

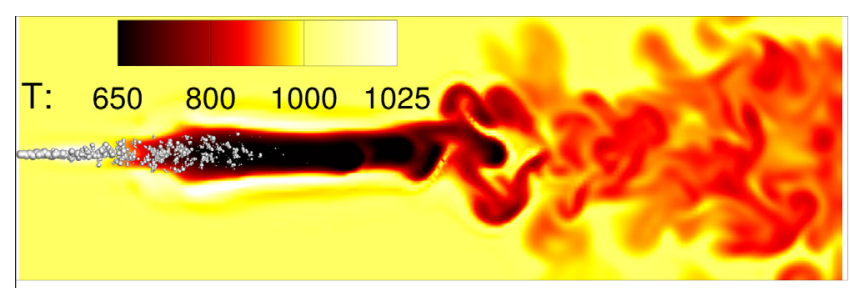

(a)

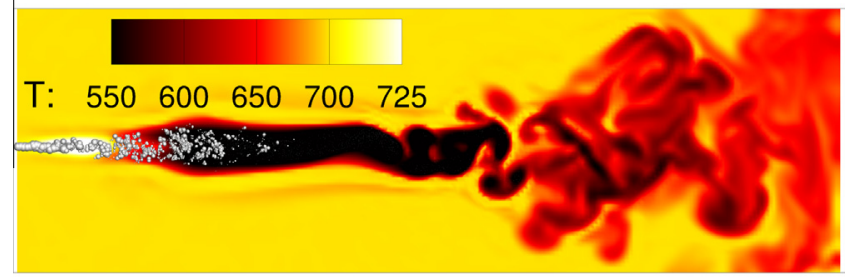

(b)

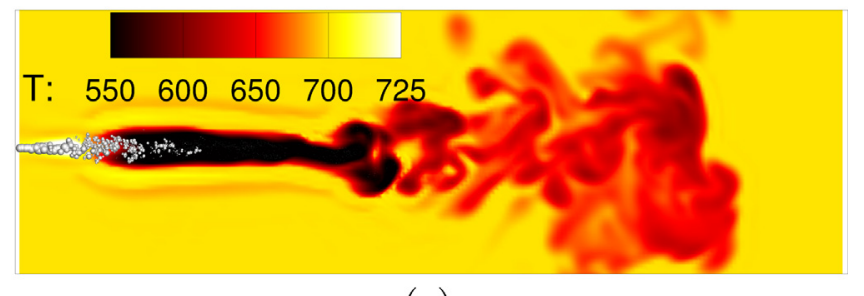

(c)

Fig. 15. Contours of the gas temperature $(K)$ in the spray chamber for different gas densities and temperatures at $t=2.0 \mathrm{~ms}$ : (a) $T=1000 \mathrm{~K}, \rho=14.8 \mathrm{~kg} / \mathrm{m}^{3}$, (b) $T=700 \mathrm{~K}, \rho=14.8 \mathrm{~kg} / \mathrm{m}^{3}$, (c) $T=700 \mathrm{~K}, \rho=30.0 \mathrm{~kg} / \mathrm{m}^{3}$.

flow overlap the regions with high concentration of fuel vapor and thus hindering further evaporation of droplets. These observations are confirmed with the conditional gas flow variables plotted as a function of droplet diameter in Fig. 7.

As indicated before, for lower initial gas temperature of $700 \mathrm{~K}$, small droplets survive at the tip of the spray for the low chamber temperature, where the carrier gas is highly saturated by the fuel vapor and has significantly lower temperature. The "potential core" length of spray-induced gas jet, defined to be the distance between the nozzle and the point where the main gas jet starts to "break", is the determining factor in the prediction of spray liquid length at lower gas temperature of $700 \mathrm{~K}$. When the main 
gas jet breaks to smaller turbulent flow structures and the dispersion and mixing increases, the regions around droplets are fed with fresh unsaturated higher energy gas and as a result the droplet evaporation quickly increases. At the same time, the spray generated turbulent flow transports and mixes better the surrounding hotter gas into the spray region. This also enhances the evaporation and shortens the liquid penetration. The evaporation also cools down the surrounding gas, leading to slowing down of the droplet evaporation and higher liquid penetration. As a result of these two competing mechanisms, the location of the core gas jet transition to turbulence fluctuates in time, causing a significant, high amplitude (up to about $5 \mathrm{~mm}$ ) oscillations in the liquid penetration length in the lower gas temperature case (Fig. 13). As finer droplets are produced along the axis of the spray, the added mass to the gas due to the evaporation becomes significant. This further augments the induced gas momentum together with the droplet drag force and creates a high speed vapor-saturated, low-temperature gas jet with considerable density difference with the surrounding gas. The main features of the spray-induced gas jet is similar at the higher gas temperature of $1000 \mathrm{~K}$, yet the gas surrounding liquid droplets has so much thermal energy that the atomized droplets evaporate quickly and their residence times are comparatively shorter. This causes the droplets to disappear at shorter distances from the nozzle than the spray-induced gas jet transition point. Thus, the interactions between the droplets and the gas turbulence far away from the nozzle, as described above for $T=700 \mathrm{~K}$, is virtually non-existent and as a result, the fluctuations in liquid spray length is less significant (Fig. 13).

Fig. 16 compares the azimuthally averaged mean axial velocity for cases with different initial chamber conditions at the normalized distance of $x=15 d_{\text {noz }} \sqrt{\rho_{l} / \rho_{g}}$ from the injector and at time $t=2.0 \mathrm{~ms}$. This axial location is within the induced gas potential core for all simulated cases. As the main sources of momentum transfer from the spray to the gas are droplet drag force and evaporation, the mean induced velocity in the gas represents the net effects of these two important processes. The higher evaporation induces faster gas flow and the higher induced gas velocity prevents the saturation condition and leads to higher evaporation. Therefore, the evaporated vapor mass fraction and the induced gas velocity reach to a sort of equilibrium condition that depends on the gas and spray conditions. The mean spray-induced gas velocity profiles for different gas temperatures show that the net effect of droplet dynamics and added vapor mass are not very different at different gas temperatures. On the other hand, the relatively lower mean induced gas velocity at the higher gas density confirms the significantly more carrier gas resistance in the denser gas. At the same time, the narrower radial profile of velocity indicates less radial dispersion of the droplets and higher velocity gradients in the gas flow. Furthermore, the more pronounced negative mean axial velocity for the higher gas density implies the presence of augmented recirculation zones at the periphery of the spray and gas jet flow.

Fig. 17 compares the azimuthally averaged axial velocity variance at different non-dimensional axial locations for different chamber temperatures and densities. It should be noted that the variances in this figure are computed from the resolved velocities. It is shown in Fig. 17 that within the gas jet potential core, the perturbations in the gas jet are larger for the spray injected into hotter gas. Even though the average liquid evaporation is similar for sprays injected into different gas temperatures, as nearly identical mean vapor concentrations and induced gas velocities in Fig. 18a suggests, the local evaporation rate is still higher at higher initial gas temperature. At higher temperatures, the number of surviving droplets at each axial location is lower. This helps faster evaporation of incoming, freshly born droplets by decreasing the local

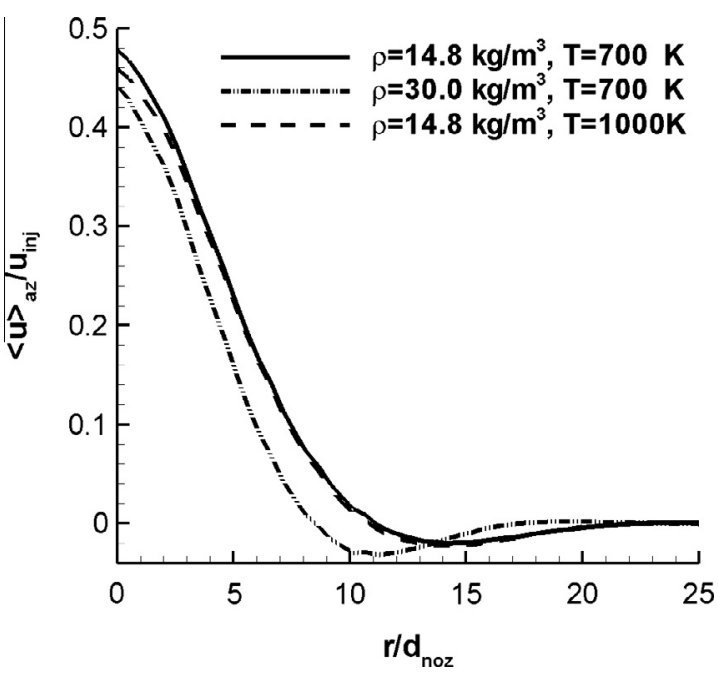

Fig. 16. Effect of chamber conditions on azimuthally averaged induced gas velocity at non-dimensional distance of $x=15$ from the injector.

vapor concentration, and causes higher velocity fluctuations in the gas jet. Even though the velocity fluctuations are significantly higher for the higher gas temperature at the shorter non-dimensional distance of $x / d_{\text {noz }} \sqrt{\rho_{l} / \rho_{g}}=15$ from the injector, they grow much less when the fuel is sprayed into hotter gas and eventually become comparable or even smaller than those computed for the lower gas temperature at $x / d_{n o z} \sqrt{\rho_{l} / \rho_{g}}=40$ and 50. At higher gas temperature of $T=1000 \mathrm{~K}$, due to disappearance of droplets and the shorter liquid penetration length, droplets which are the sources of gas flow vanish at a shorter distance. Therefore, the velocity variances begin to decrease by the transfer of energy to smaller turbulent scales. In contrast, the induced gas jet has a longer potential core and higher kinetic energy at lower gas temperature of $T=700 \mathrm{~K}$. Therefore, the growth in turbulence intensity is higher at $T=700 \mathrm{~K}$ in comparison to that seen for $T=1000 \mathrm{~K}$. The spray induced gas flow and turbulence are also functions of the initial gas density; overall the spray generates stronger flow in a less dense gas but the effect on the gas turbulence is somewhat complex. While the mean induced gas velocity in the main gas jet core (e.g. $x / d_{\text {noz }} \sqrt{\rho_{l} / \rho_{g}}=15$ ) is lower in the higher gas density case, the gas velocity perturbations are actually more significant in this case. The gas flow in the denser environment becomes turbulent at a shorter distance from the injector. However, due to shorter penetration of droplets, the main source of gas flow vanishes and the gas velocity variance decreases. Integration of $Q_{n}$ in the cross-spray planes confirms the qualitative observation in Fig. 12b and $\mathrm{c}$ that the small turbulent structures in higher gas density case grow in such a way that very far from the injector (e.g. $x / d_{\text {noz }} \sqrt{\rho_{l} / \rho_{g}}=50$ ), they become comparable with those seen in the lower gas density case.

The effect of ambient gas density on the spray-induced gas flow is better explained by considering the droplet evaporation at different gas densities. Fig. 18b shows the radial profiles of the mean evaporated fuel mass fraction and the evaporation rate parameter, $\beta=-\frac{3}{2} \operatorname{Pr} \tau_{s t} \dot{m}_{p} / m_{p}$ at $x=15 d_{n o z} \sqrt{\rho_{l} / \rho_{g}}$ for the two gas densities of 14.8 and $30.0 \mathrm{~kg} / \mathrm{m}^{3}$ at $T=700 \mathrm{~K}$. Evidently, the evaporation rate is lower in the denser gas around the spray axis but it becomes higher away from this axis due to relatively lower vapor concentration. The lower vapor concentration in this region of the flow is due to the augmented recirculation in the denser gas flow. Radial profiles of the evaporation rate parameter and axial velocity fluctua- 

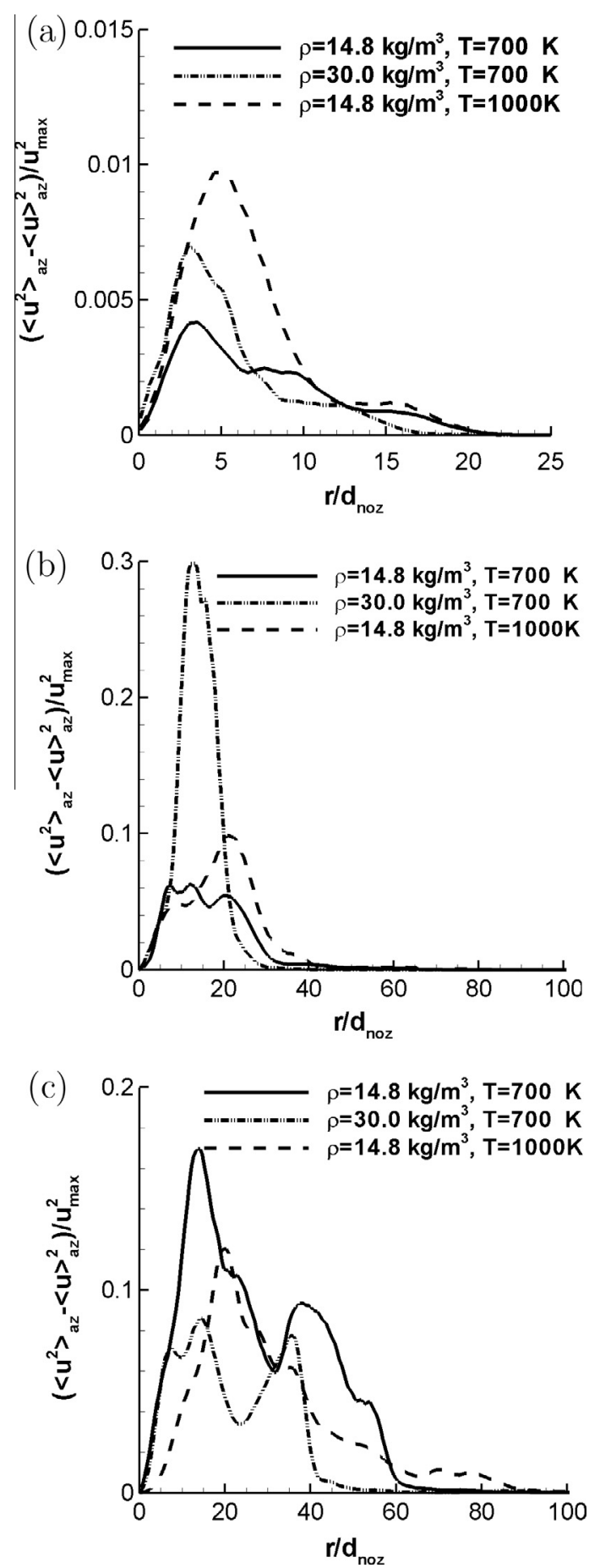

Fig. 17. Effect of chamber conditions on axial velocity fluctuations at different nondimensional distances from the injector. (a) $x=15$, (b) $x=40$, (c) $x=50$ tions show that despite the lower local evaporation (and lower mean gas axial velocity), the velocity fluctuations are higher in the denser gas. On the other hand, Fig. 19, which shows the radial variations of the root mean square (r.m.s.) of gas density, indicates that the higher velocity fluctuations in the denser gas is a result of higher density fluctuations. The momentum $\left(\rho u_{i}\right)$ change in the radial direction is dependent on the evaporation as suggested by the continuity equation. Due to lower evaporation rate in the However, due to lower radial dispersion of droplets caused by more gas resistance, the radial variation of vapor mass fraction is denser gas, the radial gradient of momentum should be smaller.
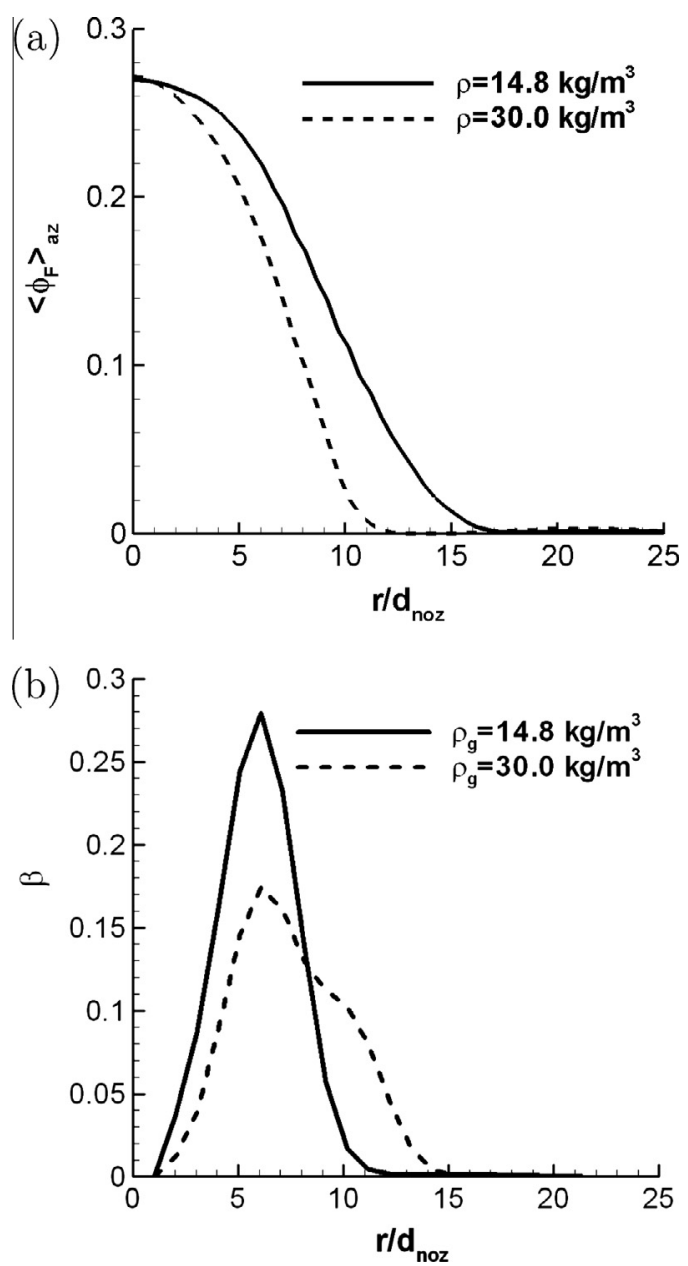

Fig. 18. Effect of gas density on radial profiles of (a) azimuthally averaged vapor fuel mass fractions and (b) non-dimensional evaporation rate at non-dimensional distance of 15 from injector at gas temperature of $700 \mathrm{~K}$.

becoming higher in denser gas. The higher vapor mass fraction gradients cause higher density gradients and higher density variance or r.m.s. in the gas flow. This is a direct result of fluctuating vapor emanating from droplets. The velocity field responds to density fluctuations in the flow to keep the mass and momentum balanced. This pushes the induced gas jet to become turbulent at a shorter distance from the nozzle such that the corresponding liquid penetration length, which correlates with the induced gas jet, to become shorter in denser gas when the gas temperature is $700 \mathrm{~K}$, as also observed in the experiment (Siebers et al., 1998).

\section{Effects of spray parameters}

In previous sections we discussed the spray behavior, the gas flow and the turbulence generated by the spray in different gas chamber conditions. However, the spray parameters also have significant effects on the spray and its interactions with the gas flow. In this section, we consider two of the most important spray parameters, i.e. the nozzle diameter and the injection pressure.

\section{Spray behavior for different nozzles}

Fig. 20 shows comparison of liquid penetration lengths as predicted by LES with the experimental data for nozzles with different diameters at various gas densities and temperatures. As mentioned before, the LES grid resolution was chosen to be about twice of the nozzle diameter in these simulations. It can be seen in Fig. 20 that 


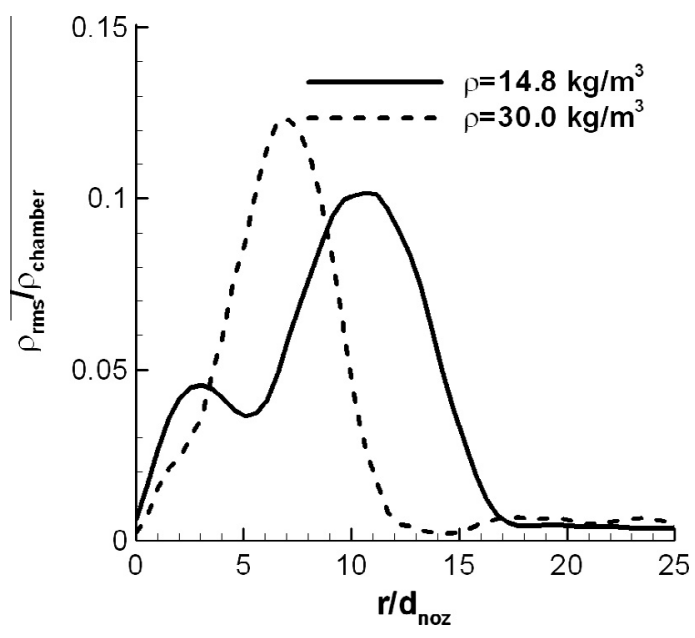

Fig. 19. Effect of chamber gas density on radial profiles of density fluctuations at non-dimensional distance of 15 from injector at gas temperature of $700 \mathrm{~K}$.

the simulated results agree fairly well with the experimental values at all conditions, confirming the overall accuracy of LES results presented in this work. While the general trend is well-captured by LES, the predictions become less accurate and the liquid penetration is under-predicted at $T=1000 \mathrm{~K}$ and over-predicted at $T=1300 \mathrm{~K}$ by LES. This is mainly due to supercritical condition for some of the droplets and lack of a reliable supercritical submodel in the present LES calculations (Irannejad and Jaberi, 2014). The supercritical effect on the evaporation is more pronounced for larger nozzles and higher liquid mass flow rates as the overall drop size, breakup and evaporation rate are dependent on the nozzle size. The higher liquid mass flow rate in sprays with larger nozzles almost proportionally increases the penetration length. With the simple model used for the dense spray region, it has been shown that the total entrained gas to the spray increases linearly with the nozzle diameter, while the injected mass flow rate increases with the square of the nozzle diameter (Siebers et al., 1999; Naber et al., 1996).

Fig. 21 shows the azimuthally averaged induced gas velocity and its fluctuations at the non-dimensional distance of $x / d_{\text {noz }} \sqrt{\rho_{l} / \rho_{g}}=15$ from the injector for the two nozzle diameters of 0.100 and $0.246 \mathrm{~mm}$. The chamber gas density and temperature

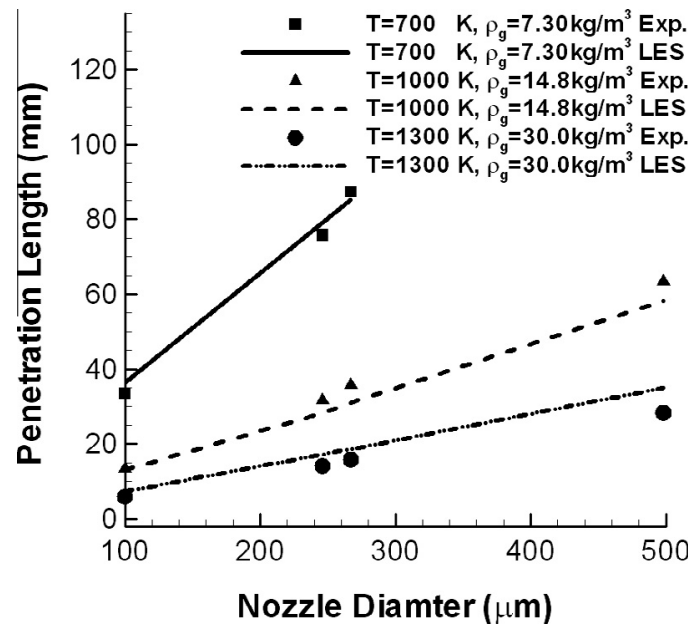

Fig. 20. Effect of nozzle diameter on the liquid penetration length at different ambient gas conditions. are $14.8 \mathrm{~kg} / \mathrm{m}^{3}$ and $1000 \mathrm{~K}$, respectively. While the normalized mean gas flow velocities for the two different nozzles are nearly identical, the variance of the gas flow velocity fluctuations generated by the larger nozzle spray is much more significant. Fig. 22 shows that the evaporation rate of small droplets (which are responsible for most of evaporation in the spray) are also higher in the case with larger nozzle. However, when scaled based on the nozzle diameter, the radial profile of normalized mean gas velocity does not change with the change in nozzle diameter, arguably because the details of spray and droplet field does not seem to have a very significant effect on the mean gas flow. Accordingly, the mean fuel vapor concentration plots are also similar when normalized in the same way. On the other hand, the higher rate of evaporation for the larger nozzle creates more perturbations in the gas velocity and lead to more significant turbulence. The higher evaporation rate of small droplets in the larger nozzle case is attributed to higher gas entrainment and lesser dissipation of the velocity and vapor gas fluctuations.

Spray behavior for various injection pressures

Fig. 23 shows the LES and experimental values of the spray length or liquid penetration length for different injection pressures and various gas temperatures and densities. The injector nozzle diameter is $100 \mu \mathrm{m}$ in all cases. Overall the LES results are in good agreement with the experimental data. The slight discrepancy in results at the lower gas temperature of $700 \mathrm{~K}$ and lower gas density of $7.3 \mathrm{~kg} / \mathrm{m}^{3}$ could be due to uncertainties in the injected liquid
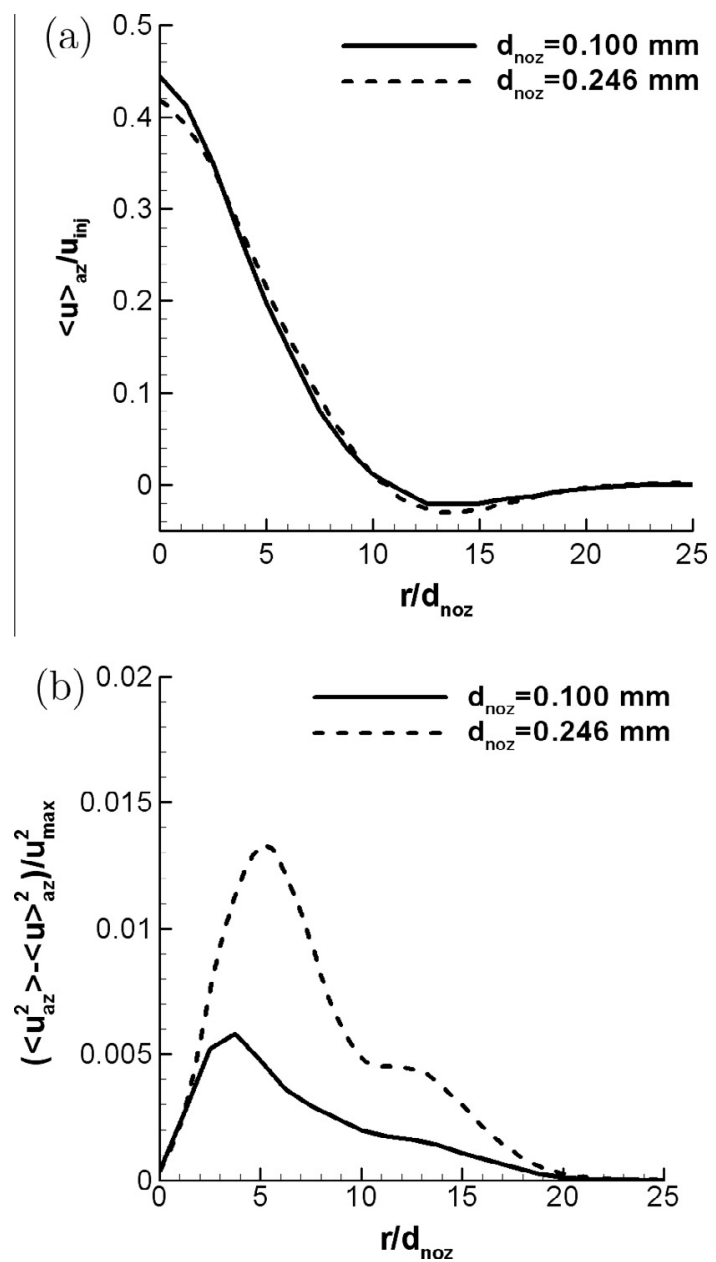

Fig. 21. Effect of nozzle diameter on (a) mean induced gas velocity, and (b) variance of induced velocity at non-dimensional distance of 15 . 


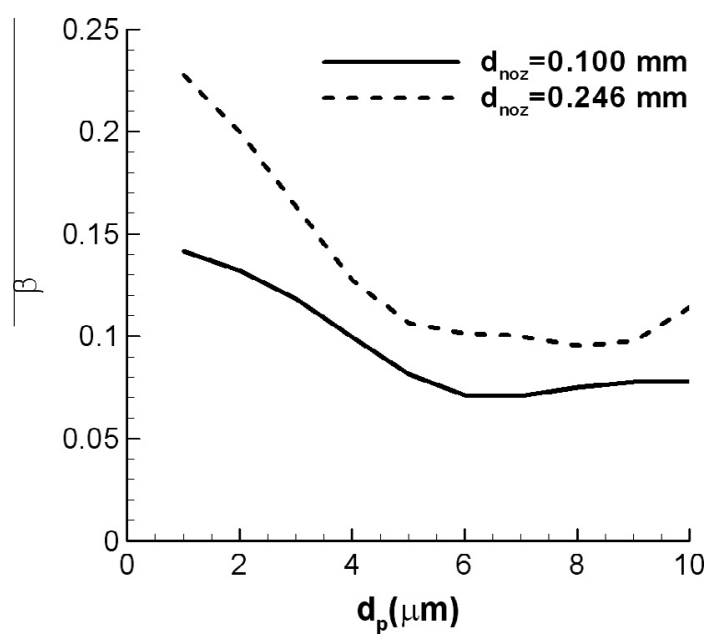

Fig. 22. Effect of nozzle diameter on the evaporation rate of small droplets.

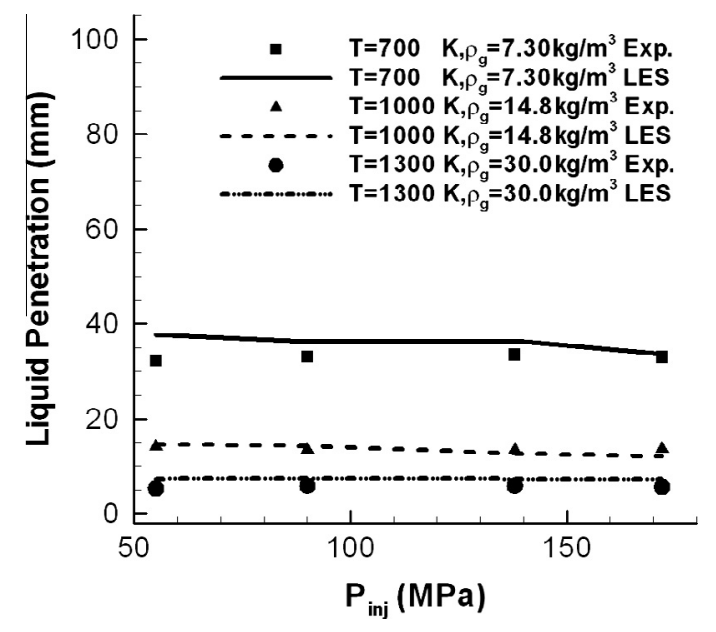

Fig. 23. Effect of the liquid injection pressure on spray penetration.

fuel conditions. More specifically, there is a considerable level of uncertainty in the velocity discharge coefficients for different liquid injection pressures as for most cases the experimental values are interpolated from the measured data for injection pressures of 72 and $138 \mathrm{MPa}$. The insensitivity of the liquid penetration length to the injection pressure in Fig. 23 suggests that the change in the fuel flow rate (caused by the injection pressure) lead to the same change in the overall fuel evaporation rate and even though the increase in injection pressure accelerates the atomization as well as the evaporation, the time required for the injected fuel to break and evaporate remain to be nearly proportional to the injection pressure.

Fig. 24 shows the mass averaged velocities of the spray droplets relative to the gas along the axis of the spray for different injection pressures at the lower gas temperature of $700 \mathrm{~K}$. As expected, the predicted relative velocity is higher along the spray axis for higher injection pressures. Higher relative velocity enhances both the breakup and the evaporation of the liquid. However, the injection pressure does not significantly change the SMD profile as shown in Fig. 25. Kamimoto et al. (1987) experimentally studied the effect of injection pressure on non-evaporating sprays and showed that with an increase in injection pressure, SMD decreases. As indicated by Kamimoto et al. (1987), this trend seems to be valid in the range of low injection pressures. However, the SMD change with the injection pressure was found to be insignificant at sufficiently high

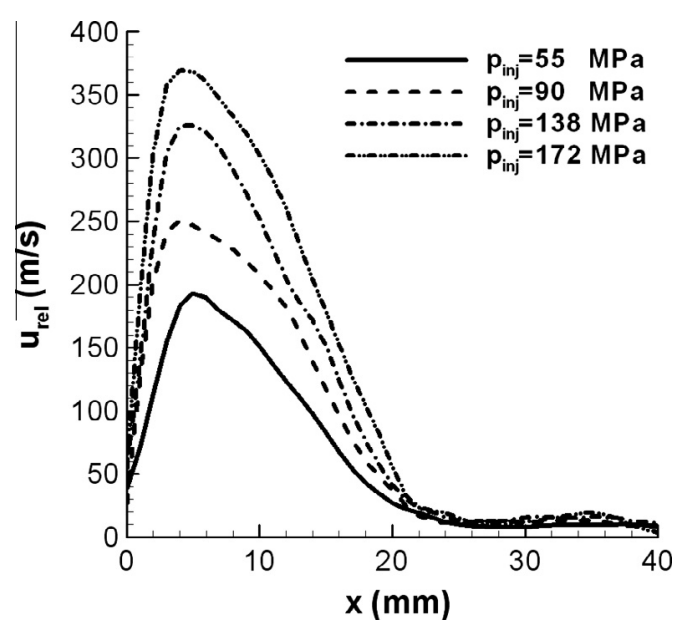

Fig. 24. Effect of injection pressure on mass-averaged relative velocity in the spray injected to the gas with $\rho=7.3 \mathrm{~kg} / \mathrm{m}^{3}$ and $T=700 \mathrm{~K}$.

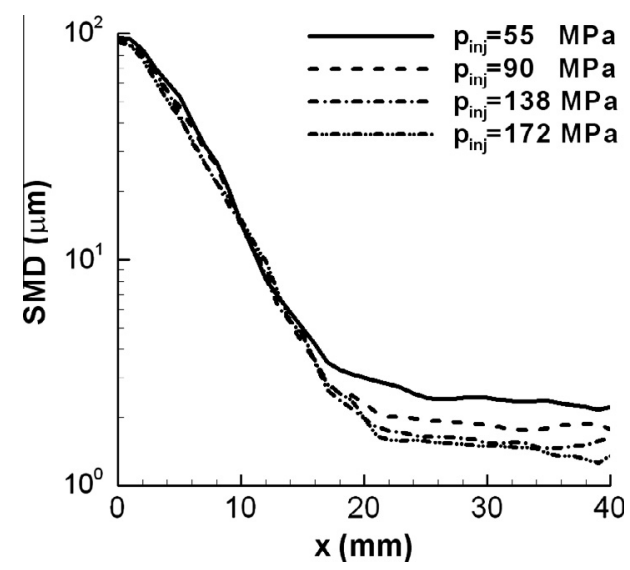

Fig. 25. Effect of injection pressure on SMD in the spray injected to the gas with $\rho=7.3 \mathrm{~kg} / \mathrm{m}^{3}$ and $T=700 \mathrm{~K}$.

injection pressures. Consistently, for the high pressure evaporating sprays considered in this paper, the effect of injection pressure on the spray is not very significant. At higher injection pressures, a more significant number of finer droplets are produced along the spray axis. But these fine droplets have very high relative velocities and evaporate faster so that the SMD does not change so much with the injection pressure. It might be noted however that at the tip of the spray, the size of surviving droplets is smaller at higher injection pressures.

Fig. 26 shows the azimuthally averaged axial velocity and its variance for different injection pressures at the non-dimensional axial distance of 20 from the injector. The azimuthally averaged vapor mass fraction and evaporation rate parameter are shown in Fig. 27. Sprays with higher injection pressures generate higher gas velocity as expected, but when the mean induced gas velocity is scaled with the corresponding liquid injection velocity, the gas velocity profiles become almost identical. In contrast, the peak normalized velocity at the spray centerline is changing non-linearly with the injection pressure. The peak values of the vapor mass fraction are also changing non-linearly with the injection pressure even though the overall (or average) amount of evaporated liquid seems to be independent of the injection pressure. The velocity perturbations in the vapor saturated gas is also changing in a similar way with the injection pressure. Understandably, the change in gas velocity variance is directly proportional to the local rate of 

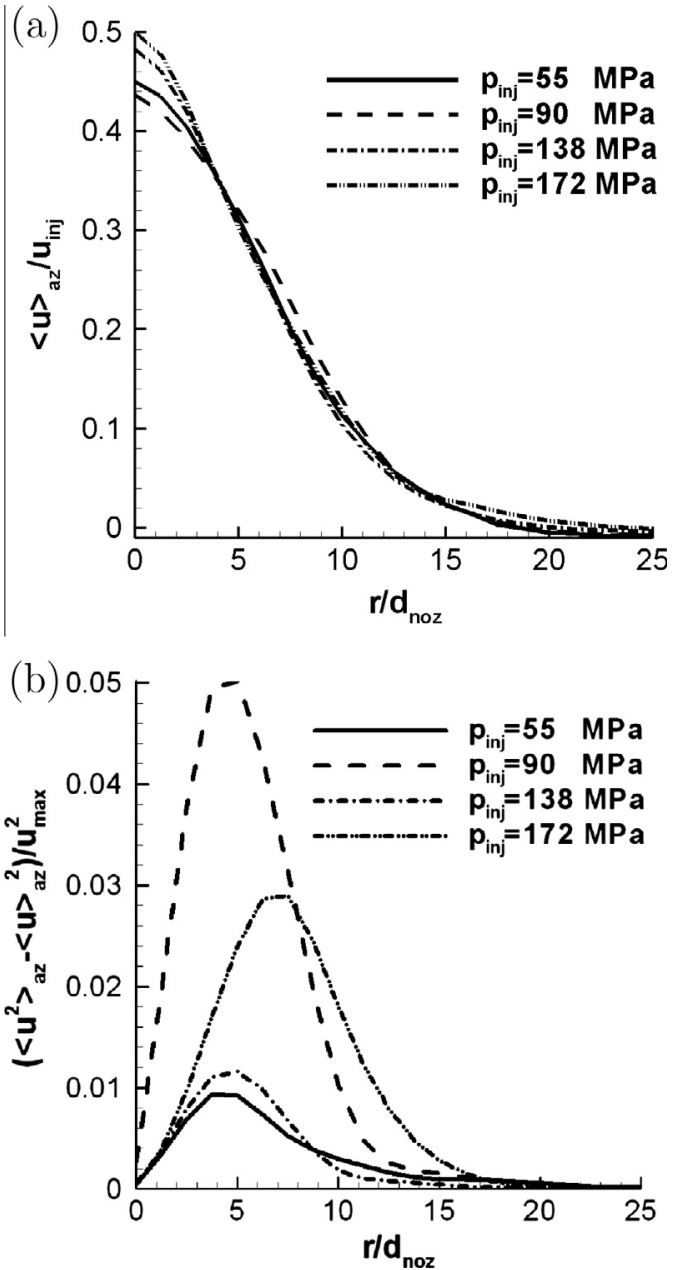

Fig. 26. Effect of injection pressure on (a) mean induced gas velocity and (b) variance of induced velocity at non-dimensional distance of $x=20$ for the gas with $\rho=7.3 \mathrm{~kg} / \mathrm{m}^{3}$ and $T=700 \mathrm{~K}$ evaporation parameter. The change in local evaporation rate is due to convection of the evaporated fuel vapor by the spray induced gas flow. An increase in injection pressure causes a higher relative velocity between phases on average, and the enhancement of droplet drag force, the evaporation, and as a result the gas velocity fluctuations. At the same time, the higher induced gas velocity more efficiently convects the evaporated fuel away from the spray and droplets, allowing higher evaporation rate to be achieved. This process becomes much more significant when the injection pressure increases from 55 to $90 \mathrm{MPa}$ and the local evaporation rate increases by an order of magnitude. As a result, the vapor mass fraction in the gas increases by the injection pressure, most notably at the spray centerline. However, due to increase in gas density, the mean normalized induced gas velocity slightly decreases (although the actual velocity value increases). With a further increase in the injection pressure from 90 to $138 \mathrm{MPa}$, the gas will be saturated to such an extent that the local evaporation rate starts to decrease. Consequently, the vapor mass fraction remains nearly the same as liquid injection pressure increases from 90 to $138 \mathrm{MPa}$. With almost the same vapor distribution, the mean normalized induced gas velocity increases as injection pressure increases. Simultaneously, there is a decrease in the evaporation rate and the gas velocity fluctuations. However, with even more increase in the injection pressure from 138 to $172 \mathrm{MPa}$, the induced gas velocity and vapor convection becomes so significant that as a result of enhanced vapor convection, the local evaporation rate increases again. This is due to the fact that the interphase momentum
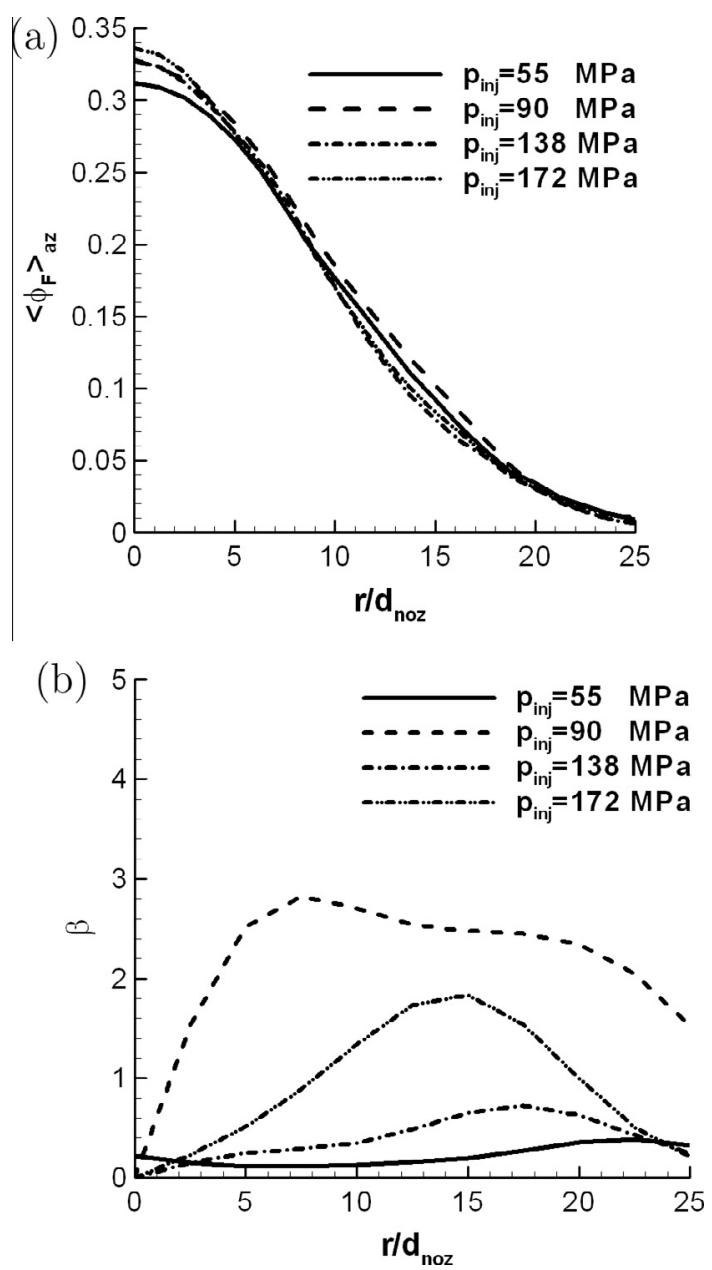

Fig. 27. Effect of injection pressure on the radial profiles of (a) the azimuthally averaged vapor fuel mass fractions and (b) the mass averaged non-dimensional evaporation rate at non-dimensional distance of 20 from the injector and gas density and temperature of $7.3 \mathrm{~kg} / \mathrm{m}^{3}$ and $700 \mathrm{~K}$, respectively.

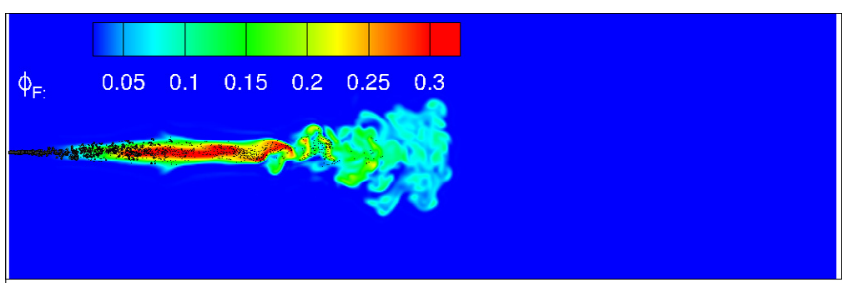

(a)

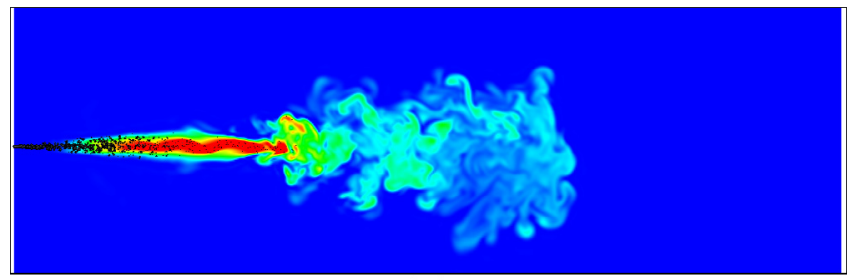

(b)

Fig. 28. Vapor contours for sprays injected with different pressures into the gas with $T=700 \mathrm{~K}, \rho=7.3 \mathrm{~kg} / \mathrm{m}^{3}$ at $t=1.0 \mathrm{~ms}$. (a) $p_{i n j}=55 \mathrm{MPa}$, (b) $p_{i n j}=172 \mathrm{MPa}$.

transfer is caused not just by the evaporation, but also by the droplet drag force. In other words, although the rate of evaporation may decrease or increase with the injection pressure, the droplet effect on the gas force monotonically increases with the injection 
pressure. This causes the induced gas velocity to increase, regardless of the rate of evaporation.

The higher induced gas velocity at higher injection pressure causes more vapor penetration, while the liquid penetration remains the same. This is evident in Fig. 28, where the contours of vapor concentration for two injection pressures of 55 and $172 \mathrm{MPa}$ are compared at $t=1.0 \mathrm{~ms}$. The fluctuations in the gas jet grow accordingly so that the mean length of the potential core of high speed, low temperature, vapor-saturated, gas jet remains nearly unchanged with the change in injection pressure. Even with the same mean gas potential core length, the higher fluctuations in the gas jet enhance the turbulence production, dissipation and mixing. As suggested by the results in Fig. 28 and consistent with the observation that the liquid penetration does not change so much with the injection pressure, the earlier distribution of evaporated vapor does not change with the injection pressure.

\section{Summary and conclusions}

Large eddy simulations of high speed evaporating sprays with significant spray-induced gas flow turbulence are conducted with a hybrid Eulerian Lagrangian mathematical/computational methodology. The spray, modeled with Lagrangian droplets, stochastic breakup and non-equilibrium heat and mass transfer models, is fully coupled with the gas phase flow and turbulence through mass, momentum and energy coupling terms in the resolved and subgrid-scale gas equations. The simulated sprays are issued into a quiescent heated chamber at different gas conditions. A wide range of chamber gas temperatures and densities (or pressures) as well as spray injection pressures and nozzle diameters are considered. The predicted results with the LES/Spray model are shown to compare well with the experimental data for all tested spray and gas conditions, indicating the reliability of the model. The interactions of spray with the spray induced gas flow and turbulence are studied by examining global and local flow and spray variables. It is shown that spray generates more turbulence at higher gas chamber temperatures due to higher local evaporation rate. The induced gas jet becomes turbulent at a shorter distance from the injector for a denser chamber gas because of higher velocity and density fluctuations in the gas, caused by the momentum transfer. Furthermore, the spray is shown to generate stronger perturbations in the gas when injected from a larger nozzle because of higher local evaporation caused by higher gas entrainment. Our results also indicate that the increase in injection pressure causes a non-linear change in evaporation rate of droplets because of related changes in the mean normalized gas flow. This explains the experimentally observed trend that the liquid penetration does not change so much with the injection pressure (for the range of injection pressures considered in the experiment) due to competing effects of evaporation and vapor convection. While the injection pressure does not affect the liquid penetration, the vapor penetrates more and mixes better at higher injection pressures due to higher induced gas velocity and turbulence.

\section{Acknowledgments}

1189 Q4 This work was supported by the U.S. Department of Energy under Agreement DE-FC26-07NT43278. Additional support was 1191 Q5 provided by the Defense Logistics Agency under Agreement DFARS-252232-7010. Computational resources were provided by the High Performance Computing Center at Michigan State University and the Stampede supercomputing system at Texas Advanced Computing Center at the University of Texas-Austin, funded by National Science Foundation Award OCI-1134872.
References

Crowe, C., Sommerfeld, M., Tsuji, Y., 1998. Multiphase flows with droplets and particles. CRC Press.

Sirignano, W.A., 2010. Fluid dynamics and transport of droplets and sprays Cambridge University Press.

Balachandar, S., Eaton, J.K., 2010. Turbulent dispersed multiphase flow. Ann. Rev. Fluid Mech. 42, 111-133.

Prosperetti, A., Tryggvason, G., 2007. Computational Methods for Multiphase Flow. Cambridge University Press.

Subramaniam, S., 2013. Lagrangian Eulerian methods for multiphase flows. Prog. Energy Combust. Sci. 39, 215-245.

Gorokhovski, M., Herrmann, M., 2008. Modeling primary atomization. Ann. Rev. Fluid Mech. 40, 343-366.

Shen, L., Yue, D., 2001. Large-eddy simulation of free-surface turbulence. J. Fluid Mech. 440, 75-116.

Li, Z., Jaberi, F.A., 2009. Turbulence-interface interactions in a two-fluid homogeneous flow. Phys. Fluids 21, 095102.

Herrmann, M., 2010. A parallel Eulerian interface tracking/Lagrangian point particle multi-scale coupling procedure. J. Comput. Phys. 229, 745-759.

Fox, R.O., 2012. Large-eddy-simulation tools for multiphase flows. Ann. Rev. Fluid Mech. 44, 47-76.

Moin, P., Apte, S.V., 2006. Large-eddy simulation of realistic gas turbine combustors AIAA J. 44, 698-708.

Patel, N., Menon, S., 2008. Simulation of spray turbulence flame interactions in a lean direct injection combustor. Combust. Flame 153, 228-257.

Banaeizadeh, A., Afshari, A., Schock, H., Jaberi, F., 2013. Large eddy simulations of turbulent flows in internal combustion engines. Int. J. Heat Mass Transfer 60, 781-796.

Bini, M., Jones, W.P., 2009. Large eddy simulation of an evaporating acetone spray. Int. J. Heat Fluid Flow 30, 471-480.

Bharadwaj, N., Rutland, C.J., Chang, S.M., 2009. Large eddy simulation modeling of spray-induced turbulence effects. Int. J. Engine Res. 10, 97-119.

Irannejad, A., Jaberi, F., 2014. Large eddy simulation of turbulent spray breakup and evaporation. Int. J. Multiphase Flow 61, 108-128.

Irannejad, A., Banaeizadeh, A., Jaberi, F., in press. Large eddy simulation of turbulent spray combustion. Combust. Flame. http://dx.doi.org/10.1016/ j.combustflame.2014.07.029, in press.

Hetsroni, G., 1989. Particles-turbulence interaction. Int. J. Multiphase Flow 15, 735 746.

Elghobashi, S., Truesdell, G.C., 1993. On the two-way interaction between homogeneous turbulence and dispersed solid particles. i: Turbulence modification. Phys. Fluids A 5, 1790-1801.

Mashayek, F., Pandya, R.V.R., 2003. Analytical description of particle/droplet-laden turbulent flows. Prog. Energy Combust. Sci. 29, 329-378.

Crowe, C.T., 2000. On models for turbulence modulation in fluid particle flows. Int. J. Multiphase Flow 26, 719-727.

Eaton, J.K., 2006. In: Multiphase Flow Handbook. Taylor and Francis, pp. 86-98 Chapter: Turbulence modulation by particles.

Eaton, J.K., 2009. Two-way coupled turbulence simulations of gas-particle flows using point-particle tracking. Int. J. Multiphase Flow 35, 792-800.

Chen, J.H., Wu, J.S., Faeth, G.M., 2000. Turbulence generation in homogeneous particle-laden flows. AIAA J. 38, 636-642.

Birouk, M., Gokalp, I., 2006. Current status of droplet evaporation in turbulent flows. Prog. Energy Combust. Sci. 32, 408-423.

Reveillon, J., Demoulin, F.X., 2007. Effects of the preferential segregation of droplets on evaporation and turbulent mixing. J. Fluid Mech. 583, 273-302.

Mashayek, F., 1998. Droplet-turbulence interactions in low Mach number homogeneous shear two phase flows. J. Fluid Mech. 367, 163-203.

Miller, R.S., Bellan, J., 1999. Direct numerical simulation of a confined threedimensional gas mixing layer with one evaporating hydrocarbon-droplet-laden stream. J. Fluid Mech. 384, 293-338.

Okongo, N., Bellan, J., 2004. Consistent large-eddy simulation of a temporal mixing layer laden with evaporating drops. Part 1: Direct numerical simulation, formulation and a priori analysis. J. Fluid Mech. 499, 1-47.

Leboissetier, A., Okongo, N., Bellan, J., 2005. Consistent large-eddy simulation of temporal mixing layer laden with evaporating drops. Part 2: A posteriori modelling. J. Fluid Mech. 523, 37-78.

Cochet, M., Bazile, R., Ferret, B., Cazin, S., 2009. Evaporation of polydispersed droplets in a highly turbulent channel flow. Exp. Fluids 47, 379-394.

Afshari, A., Jaberi, F.A., Shih, T.I.P., 2008. Large-eddy simulations of turbulent flows in an axisymmetric dump combustor. AIAA J. 46, 1576-1592.

Moin, P., Squires, K., Cabot, W., Lee, S., 1991. A dynamic subgrid scale model for compressible turbulence and scalar transport. Phys. Fluids A 3, 27462757.

Ghosal, S., Lund, T.S., Moin, P., Akselvoll, K., 1995. Consistent large-eddy simulation of a temporal mixing layer laden with evaporating drops. Part 2: A posterior modeling. J. Fluid Mech. 286, 229-255.

Loth, E., 2000. Numerical approaches for motion of dispersed particles, droplets and bubbles. Prog. Energy Combust. Sci. 26, 161-223.

Pozorski, J., Apte, S.V., 2009. Filtered particle tracking in isotropic turbulence and stochastic modeling of subgrid-scale dispersion. Int. J. Multiphase Flow 35 118-128.

Gardiner, C.W., 1990. Handbook of Stochastic Methods for Physics Chemistry and the Natural Sciences. Corrected, second ed.
1198

1199

1200

1201

1203

1204

1206

1207

1208

1210

1211

1212

1213

1214

1215

1216

1217

1218

1219

1220

1221

1222

122

1225

1226

1227

1228

1229

1231

1232

1233

1234

1235

1236 
Bellan, J., Harstad, K., 1987. The details of the convective evaporation of dense and dilute clusters of drops. Int. J. Heat Mass Transfer 30, 10831093.

Liu, A.B., Mather, D., Reitz, R.D., 1993. Modeling the effects of drop drag and breakup on fuel sprays. SAE 930072.

Jiang, X., Siamas, G.A., Jagus, K., Karayiannis, T.G., 2010. Physical modeling and advanced simulations of gas-liquid two-phase jet flows in atomization and sprays. Prog. Energy Combust. Sci. 36, 131-167.

Reitz, R.D., 1987. Modeling atomization processes in high pressure vaporizing sprays. Atom. Spray Tech. 3, 309-337.

Kastengren, A., Tilocco, F.Z., Powell, C.F., Manin, J., Pickett, L.M., Payri, R., Bazyn, T. 2012. Engine combustion network (ECN): measurements of nozzle geometry and hydraulic behaviour. Atom. Sprays 22, 1011-1052.

Pickett, L.M., 2013. Engine Combustion Network, Sandia National Laboratory. <http://www.sandia.gov/ecn/>.

Siebers, D.L., 1999. Scaling liquid-phase fuel penetration in diesel sprays based on mixing-limited vaporization. SAE 1999-01-0528.

Kolmogorov, A.N., 1941. On the log-normal distribution of particle sizes during breakup process. Dokl. Akad. Nauk. SSSR 31, 99-101.

Gorokhovski, M.A., Saveliev, V.L., 2003. Analyses of kolmogorovs model of breakup and its application into Lagrangian computation of liquid sprays under air-blas atomization. Phys. Fluids 15, 184-192.

Gorokhovski, M.A., Saveliev, V.L., 2008. Statistical universalities in fragmentation under scaling symmetry with a constant frequency of fragmentation. J. Phys. D: Appl. Phys. 41, 085405.

O'Rourke, PJ., Amsden, A.A. 1987. The TAB method for numerical calculations of spray droplet breakup. CONF-871142-1-Rev. LA-UR-87-2105-Rev., Los Alamos National Lab., NM.

Plich, M., Erdman, C.A., 1987. Use of breakup time data and velocity history data to predict the maximum size of stable fragments for acceleration induced breakup of a liquid drop. Int. J. Multiphase Flow 13, 741-757.
Gelfand, B.E., 1996. Droplet breakup phenomena in flows with velocity lag. Prog. Energy Combust. Sci. 22, 201-265.

Torres, D.J., ORourke, P.J., Amsden, A.A., 2003. Efficient multicomponent fuel algorithm. Combust. Theory Model. 7, 67-86.

Srivastava, S., Schock, H., Jaberi, F., 2013. Numerical simulations of turbulent sprays with a multicomponent evaporation model. SAE 2013-01-1603.

Abramzon, B., Sirignano, W.A., 1989. Droplet vaporization model for spray combustion calculations. Int. J. Heat Mass Transfer 32, 1605-1618.

Ranz, W.E., Marshall, W.R., 1952. Evaporation from drops. Chem. Eng. Prog. 48, 141146.

Siebers, D.L., 1998. Liquid phase fuel penetration in diesel sprays. SAE 980809.

Pickett, L.M., Genzale, C.L., Bruneaux, G., Malbec, L.M., Hermant, L., Christiansen, C., Schramm, J., 2010. Comparison of diesel spray combustion in different hightemperature, high-pressure facilities. SAE 01-2106.

Bardi, M., Payri, R., Malbec, L.M., Bruneaux, G., Pickett, L.M., Manin, J., Bazyn, T. Genzale, C., 2012. Engine combustion network: comparison of spray development, vaporization, and combustion in different combustion vessels. Atom. Sprays 22, 807-842.

Poling, B.E., Prausnitz, J.M., OConnell, J.P., 2001. The Properties of Gases and Liquids, fifth ed.

Sirignano, W.A., 2005. Volume averaging for the analysis of turbulent spray flows. Int. J. Multiphase Flow 31, 675-705.

Hunt, J.C.R., Wray, A.A., Moin, P., 1987. Eddies, streams, and convergence zones in turbulent flows. Report CTR-S88, Center For Turbulence Research, CA.

Dubief, Y., Delcayre, F., 2000. On coherent-vortex identification in turbulence. J. Turbul. 1, 1-22.

Naber, J., Siebers, D., 1996. Effects of gas density and vaporization on penetration and dispersion of diesel sprays. SAE 960034.

Kamimoto, T., Yokota, H., Kobayashi, H., 1987. Effect of high pressure injection on soot formation processes in a rapid compression machine to simulate diesel flames. SAE (No. CONF-8709177-). 